\title{
Il dialetto di Agnone.
}

\section{La fonetica e la flessione.}

\section{Introduzione.}

Agnone è una piccola e ridente città al confine settentrionale del Molise. $\grave{E}$ posta sulla cresta di una collina che, staccandosi a N. E., s' avanza verso O., e scende con ripidissimo pendio da tre lati sulla conca dell' alta valle del Verrino, affluente del Trigno. Sorge quindi nel territorio del Sannio caracenio, a una decina di chilometri dal sito ove era Bovianum vetus, forse a fianco di quell' antica Aquilonia, di cui parla Livio nel X libro della prima decade.

Il popolo è composto di due elementi assai ben distinti: dei quali l' uno è formato di contadini che vivono in campagna, in villaggetti e case sparse, e vengono in città periodicamente e per breve tempo; l' altro è composto di artieri vivaci e laboriosi e di borghesi professionisti, che hanno tra loro stretti vincoli d' interessi, di amicizie e parentele; in modo che si puó dire non esista una borghesia veramente distinta dal ceto degli artigiani. Di qui due correnti dialettali: l' una fa capo ai contadini, l'altra ai cittadini; e mentre quella sviluppa i caratteri del dialetto, evolvendoli, questa raffrena e arresta la tendenza evolutrice.

La posizione geografica ed etnica della città si riflette sul dialetto, che, movendo dalle parlate del Molise, da una parte dà la mano ai dialetti dell' alta valle del Volturno, dall' altra si connette intimamente con quelli del Chietino e del basso Aquilano, onde spinge lo sguardo al Teramano; lungi poi tien l'occhio alle terre del basso Foggiano e del Barese.

Fonte scritta è il Vocabolario del dialetto agnonese del dott. Giuseppe Cremonese (Agnone-Bastone 1893). L' autore era di Agnone; e, per l' esercizio della sua professione di medico, era in continuo e diretto contatto col popolo. A lui quindi non mancò il modo di avere esatta notizia delle voci dialettali, nè l' orecchio avvezzo a percepirle; gli manco invece una preparazione conveniente. Egli non ha un concetto esatto del dialetto; non usa un sistema razionale e costante per la trascrizione dei suoni; pone la forma più volgare accanto alla più civile, senza distinguer l' una dall' altra; troppo spesso poi si serve di espedienti grafici che rendono impossibile la percezione esatta dei fenomeni. Valga un esempio per darne un'idea: egli 
adduce come articolo p. f. les, mentre è $l o$, movendo, non saprei con quale ragione, dalla trascrizione francese. Percio questo vocabolario non è un buono strumento di studio.

Il mio lavoro deve al Cremonese il primo spoglio solamente; del resto è frutto della mia quinquenne consuetudine della parlata locale, dell' aiuto paziente di amiche e amici agnonesi; ed è venuto avanti lentamente in Agnone, dove ogni nuovo dubbio poteva agevolmente esser dissipato.

\section{La Fonetica.}

Avvertenza. Uso $c$ e $g$ per le palatali, $h$ per l'aspirata sonora, $n$ $m$ per un suono né tutto consonantico nè interamente nasalizzato; oe per un suono più cupo di $\partial, \bar{u} \bar{\imath}$ per suoni pingui nelle voci neolatine.

\section{Vocali.}

\section{Vocali toniche.}

á.

a) In sillaba aperta di parossitoni o di ossitoni provenienti da parossitoni:

1. Nel volgo dà eá éa "suono lungo che comincia con $e$ e va a finire insensibilmente in $a^{4}$ (M.-L. I, 204); nelle persone più civili suona $a^{0}$ : addomeána domani, deáto jukedto dato giocato nel senso di detto fatto, hrattakeás grattugia, kambeáno, seánə intiero; 1 gl' infiniti: abballed inghiottire, arkapeá scegliere, kjoked piegare, kropeá, kromzed pensare (mistione di credo e penso). ${ }^{2}$

2. Per effetto di $u$ precedente diviene $u \phi$, e, con ritrazione d' accento, uis: abbrošú ardere e bruciare, akkskkariuó accoccolarsi, allošsuo scorgere *ad-luceare, adonu $\oint$ radunare, allakkuó gridare, apporuó appurare, arvolluó rivoltare, assokuó asciugare, kavotuó perforare, kutruó smuovere, joruó giurare, nḡokkoruó attrappirsi, fruwwó consumare, arrotoruóto rotolato, skarropuoto rovinato, sponaruóla spillato *expinula-, mosorúsmo misuriamo, mosorú misurò. ${ }^{3}$ Nei nomi questo riflesso è limitato al m. 8., determinatovi da ru il, stu questo, ssu cotesto, nu uno: ru nuosso il naso, ru puólo il palo, ssu kuóno cotesto cane, nu kuópo un capo (di filo ecc.), stu ssuómo questo sciame; e cosi ru pruóto prato, ru fruóto fratello, ru wuóso vaso, ru wuódo guado, servendo come utile elemento flessivo. Nelle persone più civili resta $a^{0}$.

3. Preceduto da $i, j, n, l, k j, \hat{g} j$ diventa $i e^{\prime}, j e$, , onde l' ulteriore sviluppo volgare $i j o$ (cfr. D' Ovidio, AG. IV, 247): kjiéno piano, maliéto castrato, kualiẹto quagliata, piẹco piace, mbriéko ubbriaco;

1 Pel significato cfr. Ascoli AG. XV, 317 .

v. D' Oridio AG. IV, 167.

S Nell' esemplificazione si abbia presente che pure l' etimologico 0 atono dà $u$ nei dialetti meridionali, onde muove l'agnonese. 
un' estesa categoria di verbi: mañį mangiare, nkanį indicare, stokkjié scheggiare, cui son da aggiungere tutti quelli in -oj-, provenienti da -igare, -icare, *idjare: ${ }^{1}$ kammonojé camminare, arrovojé arrivare, fatojé faticare, ecc.; e regolarmente le varie forme flessive, fra le quali i participi: skuñiéta sgusciato, sbrajęto sbrigato ecc. - Sull' analogia dei participi molte altre parole in -at- hanno lo stesso riflesso: zmiéto senata, quanto cape nel zoneálo grembiale, hradiẹta gridata, vaćaniẹta vicinato, frottiéto frittata, la kustięta costato. Le persone più civili anche in questo caso rispondono con $a^{0}$. A Teramo e nel Chietino l' evoluzione fonica continua, chiudendo $i j$ in $i$ (cfr. il Savini, Dial. di Teramo, e De Lollis, AG. XII, 3 sg.).

4. Dato -i, si ha ancora ié, jé, ijo: i plurali m. kiéno cani, pięlo pali, ligiémo legami, kutatriẹr ragazzi, suldiẹto soldati ecc.; la $2^{a}$ p.s. pr. $t$ ' abbrięma sei assetato, abbiéda badi ecc. (cfr. l' arpinate in AG. XIII, 300, e l' abbruzzese in genere). Son da aggiungere le parole in -ái: fé fai, stié stai, sié sai, oramié oramai, paskriẹ *post-cras, prassié $>$ *prassai $>$ *purassai o *perassai.

b) In sillaba chiusa o in parole proparossitone:

5. Resta a: jukkdnno covata, 2 kákk qualche, kutárno chitarra, långo sete, látro, mákkja, máldo maltha, m. márko marchio, ndákks *intagica,3 kúálto caglio, kúánda, skáññ scambio, dsono, kañdávono.

6. Per effetto di $u$ precedente, e quindi di $r u, s i u, s s u, n u$ (cfr. § 2), passa in $9 ; 4$ norma cui si sottraggono buon numero di parole proparossitone: ru pǵtro il padre, ru róññ araneu graffiatura, ru ró̧ša raschio, bbusóǵrda bugiardo, ru pónna panno, ru trónto $<$ terraneu secchietto, ru fósş fascio, ru lóppo lappo, ru folgteto filaccio, ru pósso passo, ru mmósta basto, allokkóvono gridavano, allokkónno gridando. $\mathrm{E}$ da avvertire peraltro che spesso le parole che hanno - $\phi$ - prodotto da $r u$ ecc., lo mantengono pure se questo viene a mancare; anzi, prodotta per esigenze fonetiche una nuova forma pel m. s., l' analogia ha agito in modo da estenderla a tutti i m. s. di parole uscenti in -u. Degno di nota è il m. bbusuộ́rdo, oltre al s. c. bbušgórdo, e su di esso il f. bbusóǵrda, oltre al regolare bbušárda, sull' analogia delle parole con -ŏ́-. - Il ceto più civile risponde sempre con $a$.

7. Per gli effetti di $i, j, \tilde{n}, l, k j, \hat{g} j, \zeta$, precedenti e contigui,

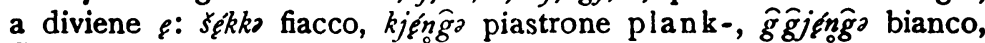
lénna ghianda, pajęlta piatto, kjend pianta; - talvolta pure per $\dot{g}$, $\dot{c} \xi, j$ non contigui, o susseguenti: félǵo falce, $k \xi \xi l g$ a calcio e calcina, $\zeta k \xi p$ pa schiappa, léməjo (nap. lamia) soffitta, vęsko vasca, Kambuwesssa Campobasso. C' è késso cassa da porvi i pettini del telaio, e qualche altra parola, in cui non vedo chiara la ragione di $-\xi_{-}^{-5} .5$

1 Per *-idjare cfr. Parodi, Misc. Asc., 457 e sg.

2 Rispetto al suffisso v. Salvioni, App. merid., p. 12.

3 Cfr. Ascoli, AG. XIV, 338.

4 Per la gran parte delle parole si potrebbe veder pure l' azione di -ŭ.

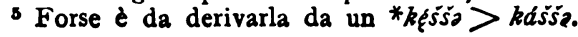


8. Per gli effetti di -I, sempre f: fjono agni i, pembono pampani, rapęcco ritagli di ostie, ténno tanni, tr fitto tratti, beffo baffi, fsono asini, nnéndo innanzi. Nella $2^{2}$ p. s. del presente $c^{\prime}$ è ié, jé, come in sillaba aperta dei parossitoni: kiémbro incammeri mangi di grasso, friébboko fabbrichi, mięrño mangi. ${ }^{2}$

9. -áriu, -ária hanno il duplice esito dei dialetti meridionali; quindi -eára m. e f.; e m. iéra, f. giro, come se venissero da -ĕ́r(cfr. $\$ \S 21$ e 22): I. kallareáro calderaio, kambaneáro, latteára, calleáro cellariu, kuatreáro ragazzo forse da * quartariu, ${ }^{2}$ ecc. II. aliéro infermiccio (da connetterc col troiano aláo sbadigliare), kjuppóira pioppaia, nəkkjgira terreno non lavorato da un anno *annicularia, fumiéro letame (cfr. Körting 3766), jurnatiéro contadino che lavora alla giornata, manóira maniera, maniér ramaiuolo *manariu, sprokgira sciupona, farzaliéra m. e farzolgira f. buffone, da fárzala buffonata, karrgiro corsa, mandgiro grembiale.

$$
\text { i. }
$$

10. In sillaba a perta di parole parossito ne riflesso originario è $\hat{z}$, che resta nelle persone più civili; nel volgo si dittonga in $o^{\prime} i$, e nei contadini $c^{\prime}$ è indizio di ulteriore sviluppo in uói ; nel ceto cittadino s' avverte éi-. $\rho i$ è normale a Cerignola e a Bitonto; per ci nel vastese cfr. il Rolin, 1. c., 12. -flis ćuvọilo ecc.; -fle mandóilo tovaglia ecc.; f́nu, -a votóina, mappóino strofinaccio, malóino, kandóina, fassş̧ina fascio; -f vu, -a kurróivo disgusto, lọivo oliva; - $\mathrm{f} \mathrm{cu},-\mathrm{a}$ arroddóiks ortica, formóiko formica; f tu, -a Margaróóito, nzalanóito scimunito, ar rakóito rauco (cfr. il troiano abbrakśto); -fre, colla caduta di -re e coll' epitesi di -jo: monójjo, murófjo morire, nójj implēre passato alla IV; -fmus drumóimo dorminus, sondóimo sentiamo; -f te, -ftis fornóito ecc.; -f́vi šọoivo uscii; così appróimo prima, conóstso * cinisia, bbušojjo ecc.

I1. In parole ossitone suona $i$ chiaro: akkuşsi così, accanto

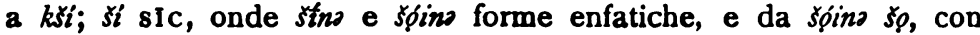
apocope di -ina; akkulli in quel modo; e gl' infiniti ajaprt aprire, moni venire ecc.

12. In sillaba chiusa e nei proparossitoni resta $i$ chiaro:

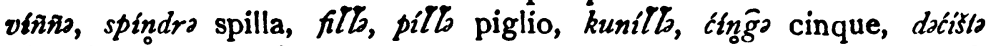
dicesti, lisko esca, liññ temperamento, maritomo mio marito, spikuoelo spigolo, aćtidora uccidere, dićora, milajo accanto a móilo mila (cfr. Bianchi, AG. XIII, 219).

Turbamenti fonetici di ragion flessiva si vedranno in seguito.

é.

C' è metafonesi conforme al tipo napoletano; quindi -i-, dati $-\mathbf{I},-\breve{u}$.

1 Cfr. il vastese in Rolin, Die Mundart von Vasto, Praga, p. 8 e 9.

2 Cfr. De Bartholomaeis, AG. XV, 353. 
a) In sillaba aperta nei parossitoni:

13. Dati -éc... a, .. e , . o, l'originario é si mantiene nel ceto civile, onde si parte con $\varepsilon i$, e attraverso il poco diffuso $\ddot{a} i$ si

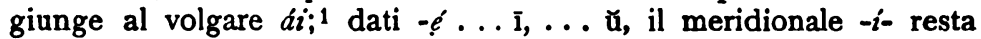
nel ceto civile con suono pingue $-i-$, onde per $e_{i}$ perviene a $\phi^{\prime} i$

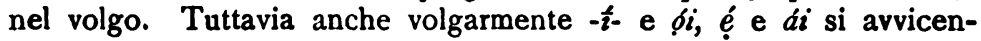
dano, e la ragione si vedrà al $\S 60$.

14. I. ćáiro cēra, kráito creta, massáiro stasera (il cui ma- è da mettere insieme con madə- di madəniídə nudo, madomeán stamane), kannáila candela, p. abbláit mistione di beta e *blitum (v. Salvioni, Ap. mer., p. 7), haśtáima bestemmia, páino pena; -énsa, -e spáiso spesa, staiso della tela, máis mese, mbáis appesa; -ére, con apocope di -re, tané, sapé, onde con epitesi di -jo il civile tanéjo ecc., e, collo sviluppo volgare in -ájji, putájjo potere, vadajjo vedere; váido vedo e vede, vodáivo vedeva. Il. šlrájjo striga, cáin cinis, sáiso ${ }^{2}$ zizza, náira nera, páiro le pere, dáito le dita; váivo bevo e beve.

15. I. aćóito aceto, trappóits trappeto, lovợito oliveto, saróina sereno, arkjọinə replēnu, pulgógin pulcino, móil melo (cfr. D' Ovidio AG. XIII, 447), p. róito reti da portar la paglia, hrọino reni; énsu, -i spóiso speso, mọ́iš mesi, mbộiso appesi, pajọišs paesi e territori; vớido vedi, póisa pesi; putassóima potessimo e potremmo, dassóima dessimo e daremmo da -ssémus. ${ }^{3}$ II. dóito dito, póira pero, nóiro nero, mandosọino grembiale, póilo pelo, rocóivo ricevo, kusira *'ccu-illu quello; vợivo bevi.

16. Le parole che si sottraggono al § 14 sono poco numerose; e di esse le più seguono le sorti di -б-, alcune di -f-. Tra le prime sono specialmente notevoli quelle in -ếria:4 mat́piro macerie (cfr. il napoletano macéçro), céîr aspetto da *cēria < cêrea. Inoltre noto nóivo neve, mmóito invece, póipo pepe, sóito sēta e sItis, pajpiss città paese, di fronte a pajaiss territorio; poi le parole s. d., come Mort́fida Mercede, Falumóin Filomena. Tra le seconde cito stróino *strēna, fọido contratto di affitto, di fronte a fóido anello nuziale, poits pietas, kugit 5 m. e f. quieto. di *vt-).

17. Al § 15 si sottrae váiro vero (cfr. il troiano víro invece

b) In sillaba chiusa e nei proparossitoni:

18. Resta $e ́$, dati -a, -e, -o: léngo lingua, famęlto famiglia, fẹsso vulva, sẹnż̇ mistione di sine e absentia, stẹlla, célto subito

1 Cfr. il vastese in Rolin, l. c., Io, Ir, e le osservazioni del Merlo, Rev. dial. rom. I, 244 .

- Se viene dal ted. zitze, come vuole il Pieri, AG. XV, 209.

8 Cfr. l' alatrino in AG. X, I69; il reatino in Campanelli, Fon., 17; e v. il campobassano e l' abruzzese.

4 Rispetto alla riduzione del suffisso -éria a *ếra attraverso *éira v. il Bianchi, AG. XIII, 239.

s Così a Scanno; v. Merlo, Rev. dial. rom. I, 415. 
cito, domenoko domenica, kuarajessomo quaresima, fémmono, mallétto $<{ }^{*}$ mal-dẹtta, f. sẹkks siccita, nétto netta; vẹngo vince, cẹ́rks cerco e cerca, célako solletico e solletica, vévoro bere, $x^{2}$ e $3^{2}$ p. facéésso, $I^{2}$ e $3^{2}$ p. mottesso ecc.

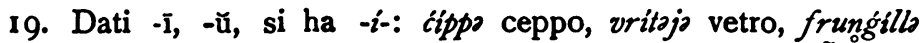
*fringillu (cfr. Salvioni, Rev. dial. I, 100-101) císto cesto, malikuoero ombelico; šilda scelto, šinda sceso, síkko secco; trídaća tredici, sídaća; 1 mitto metti, tíño tingi; la p. $6^{\mathrm{a}}$ pr. della II, III, 2 per -u- di $-\mathrm{un}(\mathrm{t})$ : vidano vedono, tiñina, vívono bevono; vodissamo vedessimo, vavissamo ecc.

20. Alcune parole sono attratte da quelle in -f-: víḉ gallinaccio, simbro semola, e parecchie voci dotte o semidotte. Alcune altre seguono le sorti di -ế-: ciérkjo ${ }^{3}$ cerchio, fiérajo feria con propagginazione di $i$ da -ria (cfr. Bianchi, AG. XIII, 238); le persone p. del perfetto vadémma, vadęsto, vadiérna, putémma ecc.; pešš non sente l' azione di $-\overline{1}$ al p. (cfr. l' arpinate in Parodi, AG. XIII, 304, e tanta parte dei dialetti merid.).

a) In sillaba aperta dei parossitoni:

21. Dati -a, -e, -o, si muove da - - - medio, mantenuto nelle persone più civili, che si sviluppa, attraverso il poco diffuso - $\{i$-, nel volgare ói: móila miele, póida piede, frọiva febbre, próita pietra, dorghito dietro; givo era, vojjjo accanto a ve viene, tójjo accanto a te tiene, lóivo levo e leva.

22. Dati -ī, -ŭ, passa in -ię-, che tende a ritrarre l' accento su -i-, onde -io-: ajéra ieri, siéro, piédo piedi; triémo tremi, tié tieni, vié vieni.

23. Intorno alla $2^{2}$ per. s. pr. ind. è da osservare che omai, tranne le forme succitate e qualche altra, parte ha -ie- e -ói-, parte, ed è la maggiore, ha -ói-: cosi priém e prọima premi, niệho e nóiho neghi, abbiẹla e abbóila covri; ma esclusivamente krọipo crepi, próiho preghi, spóira speri, lọivo levi, jóilo geli, sọiks sechi. Movendo dalle forme corrispondenti che provengono da -é-, -í-, l' analogia ha dovuto agire a pareggiare a queste anche le forme provenienti da $-\check{e}-.4$

24. -è́u, -ếa ecc. hanno regolarmente metafonesi per gli effetti di $-\overline{1},-\breve{u}$, resta $-e^{-}$per $-a,-e$; ma c' è riduzione di $*_{-i e ́}-$, per ${ }^{*}-i \partial-$,

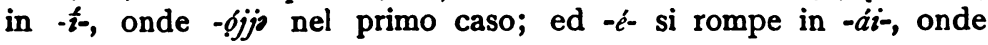

1 Lascio da parte la vexata quaestio dell' I, di trĕdecim ecc.

- Cfr. Goidanich, Misc. ling. Asc., 40I, $\$ 3$, e la minuta analisi di C. Merlo in "Gl' italiani amano, dicono ecc.", Perugia, 1908.

8 Cfr. Salvioni in Rend. ist. lom., s. II, XLI, 886.

- ẽ $<i$ è costante a Teramo per gli effetti di - $\breve{~}-\bar{i}$; l' agnonese col suo - 2 - mostra la via per cui vi si è giunti: $-i e j-<t_{0}<t$. Le $2^{e}$ p. s. agnonesi si puó fin d'ora prevedere che a non lunga scadenza non avranno più un -iẹ-. 
-ájjo nel secondo: mójj mio miei, ddojjo dio; majjo mia mie; Łgo dà pur esso jojjjo.

b) In sillaba chiusa e nei proparossitoni:

25. Dati -a, -e, -o, resta -f-: négg jo nebbia, jérvo erba, pordénźa perdita, maténna la messe, péjjo pējor (cfr. D' Ovidio, AG. X, 435, e Cocchia, Gram. lat., I1), vékkja vecchia, bbęllo bella, mannęlla manata di spighe, mandaręlla tovagliuolo, léǵga *lěvia; carvęlla le cervella, kupérkjoro i coverchi, nźźrtoro innesti; léjjo leggere, arrénno rendere, nóǵnne bruciare; faćǵnn facendo, daćgnno dicendo; ecco-qua e qua *ếcc'hoc, '́ss *essō ecco-costà e costà, éll *elllō ecco-là e cold̀. 1

26. Dati -ī, - ŭ, si hanno -ié- e -io: viẹkkjo vecchio, kummiéndo convento, assiétlo assetto delle fabbriche, bbosiesto anno bisestile, katoniéllo saliscendi, ćarviélla cervello, ciéello uccello, mandrociélla tovagliuolo rozzo, ciérvo acerbo, hurżumiẹllo ugula, mbriẹsto imprestito, kupiérkja coverchio; siẹnda senti, liẹjjo leggi, piẹnźa pensi, stiẹnno stendi; la $6^{2}$ p. della II, III: stiénnona, siéndono; jẹkkuoeto di qua, jelda di la, jeśto di costà, da *écccho-tī, *éllo-ti, *ésso-tī; doviẹllla $2 * \mathrm{de}-\mathrm{ubi}$-vellì in nessun luogo.

27. Hanno metafonesi contro il § 25: viẹnģa vengo, tiênĝa tengo, stiệnğ sto, diẹnga do. ${ }^{3}$ Piézwoela pergola forse viene da * plegula col passaggio di $-1-$ in $-i-$, ed $-\bar{e}-$ venuto a contatto di $-i-$ sarebbe è stato chiuso.

Interessante fenomeno di metafonesi è quello che si osserva nella $6^{2}$ della I coniug. a) In sillaba aperta $-\dot{\xi}$ - si chiude in - $-\dot{-}$ : trémono tremano, krépano crepano, préhona pregano, spérano sperano, lẹhono legano, nẹhono negano, lévono levano, jẹlono gelano, sẹkano secano, sékuoctana sequitano, cékzana accecano; b) in sillaba chiusa invece resta $-\dot{q}-:$ aspéittona, p'enżan pensano, $s^{\prime}$ asséttono siedono, mérdona meritano (médakana medicano, s. d.), péltonana. Così pure évana erano, di fronte al s. giva, e tutt'i pcpf.: putérano potrebbero, facéron farebbero, lajjé ron leggerebbero ecc. (cfr. \$ 45). La ragione di questo fenomeno è forse da cercare nell' analogia della II e III con. 4

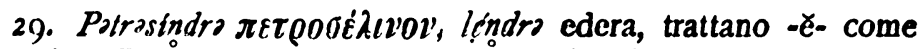
fosse -ê.-. Pel primo forse la ragione più che nell' analogia di semola, ${ }^{5}$ e da vedere nel suono stretto che aveva $\varepsilon$.

1 Vedi Merlo in Sond. Zeitschr. Gröber XXX, 445 sg., e cfr. Ascoli, AG. XV, 307 .

Vedi Merlo l. c., 452, rispetto al velli.

- Cfr. il teramano in Savini, 81 sg.; l' arpinate in AG. XIII, 302, e il toscano qua e là. La ragione è dal De Lollis cercata nell' -j- successivo, AG. XII, 21 ; il Salvioni non sarebbe alieno dal riconoscervi l' analogia della $22 \mathrm{~s}$. e $3^{2}$ pers. $p$.

4 Perchè poi per a) c' è oscuramento e per b) no, non saprei dire esattamente; forse non dev' essere estranea una certa minore sensibilità della sillaba chiusa di un proparossitono, che qua e là si può vedere in questa fonetica.

3 Così vorrebbero il D' Ovidio e Meyer-Lübke. 
ú.

30. In sillaba aperta dei parossitoni resta - thel neto civile, per turbarsi in $-\dot{i}-$, onde rompersi in $-i \dot{i} i$ - nel popolo grosso, con tendenza a ritrarre l' accento sulla prima vocale del dittongo -iu-: 1 arziuro sete, kakkjatiuro angolo formato da due rami * capulatura, krojatiuro creatura, molotiuro molitura, sarkatiuro rimendatura, kaviuto buco, iorílliuto coi capelli arruffati da cirru, con reduplicazione della sillaba iniziale, e consecutiva dissimilazione della terza (cfr. il troiano carritta), moniuto venuto, niuto empito, matiur maturo, skiura, trattiura, riuka bruco, camımariuks chiocciola, furtiuna, kiura culo, líuno, liuća, madoniudo nudo, sammíuka sambuco, síuma fiume, kjiudo chiudo ecc.

31. In sillaba chiusa e nei proparossitoni resta $-\dot{u}-$ chiaro e limpido: jünǵs giunco, ruiżo ruggine, spiindo detto di vino leggermente inacidito, f. sült lesina, m. sübblo subbio, nĝútano incudine, kúlt *cūlea (v. Pieri AG. XV, 147).

32. Portéuso è da ritenere analogo su rofọ́sa ed altre parole in -ósa, come vuole il Salvioni (Rev. dial. rom. I, 104); pónda punta, e qualche altra voce escono dai $\$ \S 30$ e $3 \mathbf{I}$.

ọ.

Base dei riflessi di -ó- son ancora i napoletani, onde l' agnonese procede rompendo il suono e dittongando.

a) In sillaba aperta di parossitoni:

33. Dati -a, -e, -o, resta - $\delta$ - nel ceto più civile, che, per -äupoco diffuso, perviene a -du-, 2 -éu- nel volgo. All' agnonese si giunge dal vastese $-d^{u}$ - (cfr. Rolin l. c. 13), che si connette al casalin. $a^{\circ} u$, ove già comincia ad avvertirsi quell' $-a$, che sarà poi normale a Teramo: $-\phi-;$; suono questo cui da Agnone si giunge più direttamente, perchè a Carovilli, distante $29 \mathrm{~km} .,-\phi-$ è costante.

-one bbonozzduno e -éuno benedizione, kambrauns e -éuna camerone (tralascio quinci innanzi la forma in -su-, che è comune a tutte le parole in -du-), coppduno ceppone, krapdun crapone, luttaun ghiottone, palanğdun mistione di falanga e pala, ${ }^{3}$ palláun,, papduns orco (a Foggia papóno, a Troia papónno), arpduns uccello di rapina, skalandráun scala a piuoli, sadauns torta con uova e formaggio, 4 Kakkaváuno Caccavone, paesello vicino, *Caccabōne; -ó́re addáuro odore, doláuro dolore, sartáuro sarto, astáuro sparviero, affruttatáur pigiatore d' uva, kuláuro colore; -a kráuno corona, patráuns padrona, láupo lupa e fame (cfr. il troiano lópo con ambo i significati); -ória ${ }^{5}$ akkurtatáuro scorciatoia, pastáuro pastoia.

1 Cfr. il dialetto di Ruvo di Puglia; e il vastese in Rolin l. c. 15.

2 Cfr. per Scanno il Merlo, Rer. dial. rom. I, 4I5.

3 V. Salvioni, Rend. ist. lomb. S. II, XL, 1049.

- Cfr. il corso fadone, in Guarnerio, AG. XIV, 156, collo stesso significato; e Salvioni, Note sarde, 84.

- Per Troia nuovo dal mio saggio (v. Stud. glott. ital. IV) e dalle mie personali notizie. 
34. Preferiscono -ęu-, pei suoni palatini precedenti e contigui (cfr. §3): porsiéuno processione, marpiéuno astuto (cfr. il s. c. arpáuno), asziẹuno azione, sẹuru fiore ecc.

35. Dati - $\breve{u},-\overline{1},-\underline{u}-$ resta nelle persone civili, e nel volgo si rompe conforme al $\S 30$. Dal teramano $-\dot{u}$ - si giunge all' agnonese attraverso il casalinc. -əü- e il vastese $\hat{\imath} u$; il quale ultimo riflesso mostra un' ulteriore evoluzione del volgare agnonese -iu-.

-ósu, -i karošliusa caro nei prezzi, pokkjiuso piagnucoloso, skakazziusa cispsoso, tartallius balbuziente; -ōriu pałšatiuro orinale, putatíuro potatoio, skalkatiuro scaricatoio, kuttiuro caldaia; niudo nodo, jîres giogo, liupa lupo, díuze due; maliuna melloni, Luttiuna ghiottoni, pakktuno grandi natiche, sartiuro sarti, siuro fiori; niurwo noi, viuwo voi; addiura tu odori.

36. Si sottraggono ai $\S 33$ e 35: sẹula sōle, m. suọla f. sẹuulo solo, rasuọ́la rasoio, parẹ́ula parola, ed alcune altri voci attratte da quelle numerose in -ŏlu, -a; ćsẹeuro cicorie, rasẹuro radimadia (cfr. innanzi rasuọ́lo), e qualche altra da $-\overline{\text { oria }}$ per ${ }^{*} \bar{o}$ ira $<{ }^{*}$ ŏria; krẹuća croce, nẹuma nome, kjẹ́uzo piove, jẹ́uzo giova, mẹurw muove, lẹuro loro.

b) In sillaba chiusa e nei proparossitoni:

37. Dati $-\mathrm{a},-\mathrm{e},-\mathrm{o}$, resta $-\dot{o}-:$ abbobkks sull' ingresso, addóndo

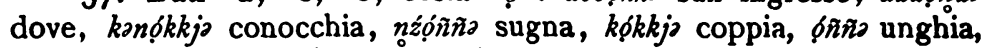
poskólla pozzanghera *pisculla (cfr. il reatino peskólla e peskóglia in Camp. 145, e accanto all' ultimo metti il troiano paskókkjo), sóltto, traskọ́rro discorrere, kóññ guscio cuneu, 1 sórǵo topo, órdono filare di viti, fóldo folta, kọ́rto corta, trộzado torbida, tọnno tonda, hrọtto grotta, pajọnzara bigonci; arpọnna riporre, oñno ungere.

38. Dati $-\breve{u},-\overline{\mathrm{i}}$, diventa $-i \mathrm{i}-$ chiaro e limpido: kjímmo piombo, fúnño fungo, lútto ranno, límmo lombo, múkkjo, rrudduilto capanna, sirkjo sorso, ssollizzo singhiozzo, zaffinno rovina, uindo lardo, tuirzo torsolo, pajünzs bigoncio, ${ }^{2}$ patilllo grassotto *patululu, trizwado torbido; díddo doti; ntiñna giungi, kanišš conosci; la $6^{2}$ della II, III: kanúsšns, uintons ungono, arpinnons ripongono.

39. Numerose parole trattano -ó- come -oó: kjuoppo pioppo, ssımuósto m. e ssjmmósto f. mosso, jauuọkkjo ginocchio, manuọkkjo covone, paduọkkja pidocchio (anche a Troia padọkkja per * ${ }^{*}\langle k-$ ), juórna accanto a jürna giorno, ${ }^{3}$ zuọlfa, vórza borsa, móvara, kjóvara (cfr. § 36), onde muọ́van ecc. Del resto il più di queste parole hanno lo stesso trattamento anche nel fiorentino.

1 Cfr. de Bartholomaeis, AG. XV, 339.

2 Facendolo derivare da *bicōngiu coll' Ascoli, AG. I, 497, n. I, e di nuovo $\mathrm{XV}, 326$.

8 Cfr. l' ital. pidokkjo, finókkjo, ginokkjo; il sic. jornu, tosc. g̨grno. 
б.

Ha metafonesi conforme al tipo napoletano.

a) In sillaba aperta di parossitoni:

40. Dati $-a,-e,-0$, resta -0 - medio nel ceto più civile, e passando per un poco diffuso -beu-, giunge al volgare -éu: 1 réuso rosa, éuwa le uova, kéura cuore, léuka là, éumo nomo, véuwa bue, sẹuro sorella; -íŏla, -ếŏla kajéulo gabbia, kapoșéulo nastro di filo, sponarẹuls fungaia di spinaruoli, vavarẹ́ulo bavaglino; nẹućo nuoce, méura muore.

41. Dati $-\breve{\mathrm{u}},-\overline{\mathbf{1}}$, passa in $-u o ́-$, che nel volgo tende a ritrarre l' accento sul primo elemento, onde -üo-. A Teramo e in molta parte d'Abruzzo l' evoluzione fonica, seguendo da -ís-, è giunta a

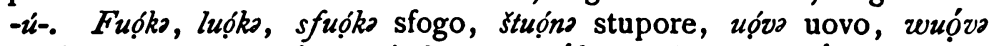
buoi, kuọ́ra cuori; -íólu, éŏlu kruñuọlo corniolo, fašuọla fagiuolo, lonziuóra lenzuolo, paparuọla peperone, sponaruóla fungo di spini; muóor muori; dapuó da *de-póst $<{ }^{*}$ depós $<{ }^{*}$ depói dopo, forma atona corrispondente pó poi; su dapuó forse kummuó quomodo?.

b) In sillaba chiusa e nei proparossitoni:

42. Dati -a, -e, -o, resta - - -: kumbósto frutta sott' aceto, f. pašş́t membro virile dei bambini, f. stózza tozzo di pane, sọcaro suocera, grtara orti, lǵkaro luoghi; artǵrćo ritorcere, arkǵrdo ricordo ricorda, pórto porto, vómmoks vomito.

43. Dati $-\breve{u},-\bar{i}$, passa in $-u \phi o-$, che nel volgo tende a ritrarre l' accento, onde -ús: akkruóş̧̧ scroscio, akkuóśto accanto, katafuórkjo *catafor(i)culu bugigattolo, kuórajo cuoio, hruósso grosso, pašuóttlo ghiacciolo, puọrko porco, skuọ́rno scorno, skuọrto guscio, skuọrdo dimenticatoio, šdussso a schiena, stramuórto strambotto con etimologia popolare, suónno sonno, taluórno seccatura, uósso usso, nddorkuóšş *intercoxeu, ruótolo rotolo, misura di peso, vruókkalo broccolo, Fuф́rlo Forli, uójjo oggi; duórma dormi, puórto porti; la $6^{2}$ della II e III duórmana dormono, artuórtano ritorcono.

44. Contro il $\S 42$ hanno metafonesi: wuólt voglio, wuórojo borea, pruóppjo proprio avv., kuókkla palla, s. fuọrćovo forbici, e qualche altra voce. Per wuóllo e wuóoraje 3 -u- è da credersi prodotto dal $w$ - precedente; ${ }^{4}$ pruópojo sarà è il maschile assunto in funzione avverbiale; kuókklo e fuórcóvo han da esser tratti dal plurale.

1 Cfr. il vastese in Rolin, I2-I3 1. c., e Merlo, Rev. dial. I, 244.

Cfr. il reatino in Campanelli, 34.

Il De Lollis vorrebbe riconoscervi l' azione di $-j$-postonico, v. AG. XII, 26 sg.

- C' $\mathrm{e}$ pure ad Arpino (v. Parodi, AG. XIII, 305; e vedi il vastese prupijo in Rolin 1. c., 13. 
45. La I coniug. ha una metafonesi incipiente nella $6^{2}$ dell' ind. pr. in sillaba aperta: a) jọkano giocano, sọnons suonano, tróvono trovano; di fronte a b): arkórdono ricordano, pórtono, sónnono sognano, vómməkano vomitano. Cosi fórono sarebbero (cfr. § 28).

46. Una cospicua serie di parole trattano -ŏ- come -ó-: I spóñna, mólla f. molle, fóllo foglia, arrospónns rispondere, s. frónns fronda, nnaskónno nascondere, mọndo monte, pọndo ponte ecc.; II múllb m. molle, artürna ritorni, spülts spogli, arrospuinna rispondi, nnaskinna nascondi, arraspinnana rispondono ecc.

47. Tra le parole proparossitone che si mostrano insensibili a - u, $-\bar{i}$, noto lómməra, vómmoka vomito, karófono garofano, e parecchie s. d.

au.

48. Conservato con infezione labiale: láwara lauro, tázwara toro; - trattato come -ó-: káus cosa; - trattato come -ŏ́-kuólo

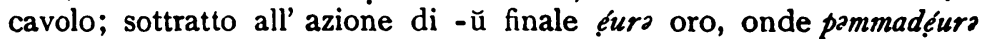
pomodoro, ñglóstra chiostro s. d.; hệudo godo, huódo godi, huọdana godono; straféuk strozzo, šrrafuọka strozzi, strafọ́kan strozzano (cfr. $§ 45$ ).

\section{Vocali atone.}

\section{Interconsonantiche.}

a) Protoniche:

49. a iniziale si conserva; nell' interno è sempre limpido e chiaro nella prima sillaba, abbastanza chiaro pure nelle altre.

50. i, e iniziali o cadono, o son sostituiti da a: I ssuóms sciame, lomósono elemosina, ñi empire, fuurándo ignorante, mbarked imbarcare, mbratteá imbrattare, mbrullie imbrogliare, muttillo imbuto; II assiémplo modello, alluštri far giorno ecc. Mediani di regola sono $-2-$; passano in $-a-$ nel futuro, condizionale e imperft. ind.:

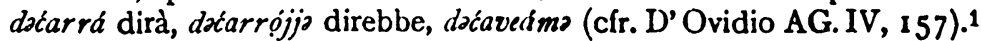
Spesso passano in $u$ nella prima sillaba a contatto di labiale: cuwwétto civetta, cuzuslęzzo gentilezza, krnweilt? cravatta.

51. o, u, a u iniziali passano in a- o cadono: I akkjięlo occhiale, atcídars uccidere, addíura odore, awánno hoc-anno, akkórro occorrere, attáuna ottone, aréfaća orefice; II spodeála, lọiva olivo, molikuoera ombelico, rẹ́kkjo orecchia, cięllo uccello. Mediano $-u-$, tuttavia nella prima sillaba spesso -a-: bambóino bon-vino specie di uva, banéuma buon uomo, kajoneáto cognato, kanớsła conoscere (cfr. Campan. 33); spessissimo -ə-: kəkọ́t́a zucca, kanókkjo conocchia, marikuoelo frutto dello spino bianco; tendenza che nel volgo è estesa quasi a tutte le parole. Anche nella seconda

1 Per analogia della I con. 
sillaba c' è di regola $-2-$, quando segue un elemento labiale 0 gutturale (v. gli esempi ai $\$ \S 2$ e 6).

b) Postoniche:

52. Tutti $i$ suoni vocalici passano in -2-; pero a di penultima s' avvicina a -2-, ma senza confondersi con esso, perchè vi si percepisce ancora una leggiera sfumatura di a. 0 , u di penultima, preceduti da gutturale, danno oe, e rigettano una sensibile infezione labiale su -k-: ákuoero aghi, fikuoero fichi, ecc.

53. - u resta nelle proclitiche $r u$ il, ssu cotesto, $s t u$ questo, $n u$ uno. - a resta, oitre che se sia pronunziato con tono enfatico, anche quando la parola sia intimamente legata alla successiva pel senso. In questo caso è sempre sensibile $1: 1^{0}$ nelle proclitiche $l a$, sta, ssa, na; $2^{0}$ nel sostantivo o aggettivo seguito da un aggettivo o sostantivo, cui sia legato: vóna véun buona buona, déla vrétto dita sporche, bbęlla citro bella ragazza; $3^{0}$ nel sing. fem. generalmente, anche se $a$ non sia etimologico, nel caso visto al n. $2^{0} ; 4^{0}$ nei numerali seguiti dal sostantivo: tręnga lọiro trenta lire; $5^{0}$ nelle forme verbali seguite da parole cui son legate pel senso: zvárda éss guarda costì; $6^{\circ}$ in tutti gl' indeclinabili congiunti ad altre parole, anche se etimologicamente $a$ non vi sia.

In iato:

54. -e-, -i-, per $*_{-i-}-*_{-i j-}$ giungono a $-j-$, onde $-2 j-2$ : $k r 2-$ jaliuro, ódojo odio, árojo aria.

55. -o-, -u-, per *u, si sviluppano in -uw-, -rw-, -əv-: Gúwánn Giovanni, vẹdavo vedova. ${ }^{3}$

\section{Accidenti generali.}

56. Prostesi frequentissima è quella di a-, che spesso tuttavia proviene da ad etimologico: abbadeá stare attento, abblaito bietola, ajéro ieri, ammujoino molestia, ammuillo molle, apprọimo prima, s' abbrowontę vergognarsi, alliéndo debole, abbórdo trina; caratteristica è in ajotd età, ausánzo usanza, ausuó usare.4

57. Epentesi di o: ssomóvoro, ssomuóstl, se non vengono da un sommovere, muovere mosso, pásama asma, affine a spasimo, pâmer ecc.; di a tra l, $r$ e una muta, o viceversa: skarapięllo scarpello, taratúffo tartufo, kalọiro ghiro. ${ }^{5}$

58. Epitesi notevole è quella di -je dopo tutte le parole ossitone: karateájo carità, ecc.; fra le quali tutti gl' infiniti divenuti ossitoni in seguito alla caduta di -re. Questo $-j o$ poi, rendendo

1 Cfr. minutamente De Lollis, Misc. Asc., 275 sg., cui si deve quest' analisi.

2 Cfr. D' Ovidio AG. IV, $157 \mathrm{sg}$.

3 Cfr. D' Ovidio 1. c., I 58 sg.

- Cfr. Meyer-Lübke R. Gr. I, 285.

- Cfr. il napoletano galiẹro. 
parossitona la parola, fa subire alla tonica interamente le leggi dei parossitoni; quindi da ni nójjj, da puté putájjo ecc. Un limite c' è, e notevolissimo: una parola, congiunta pel senso strettamente alla successiva, non ha mai -ja: nno po fea késso non può far questo, ájj' a ñni la tọina, éss a lla fóngó devo empire la brocca, costà alla fonte.

59. Gl' infiniti in -áre, -ére, -f́re perdono sempre il -re; ma quelli in Lěre lo lasciano cadere se la tonica è sillaba chiusa, non mai se è sillaba aperta: I arfọ́nno rifondere, arrł́nno rendere, arraspọnna rispondere, kjêññ piangere, éññ empire, lęjjo leggere, téññ tingere, nǵǵnna bruciare, ớñ̃a ungere, ašéñña scendere, pọ́nno far i pani, arpọnno riporre, artórćo ritorcere, sténno stendere, spánno spandere; II v'évara bere, móvara muovere, ḉdora, skrivaro, kjgivara piovere, dicara dire, métara mietere, arvivara rivivere, kjuidara ecc. 1

Qualora però -re sia sostenuto da qualche enclitica, resta: fárto farti, sondirla ecc.

60. Tutte le parole, le cui toniche hanno un sol suono vocalico nel ceto civile, e che nel volgo frangono la vocale in un dittongo, mantengono questo dittongo, se esse stanno da sole; ma, se fanno parte di un discorso, lo mantengono solo se hanno su di loro l' accento logico, o se sono in fine della frase; altrimenti il dittongo si rattrae nella vocale semplice corrispondente (cfr. Rolin l. c., p. 6). Anche le $6^{\mathrm{e}} \mathrm{p}$. fiéno fanno, viéno vengono, stiéno stanno, sięna sanno, jén hanno, puọ́n possono, wuóñ vogliono, in questo caso divengono féna, véna ecc. Esempi: ji lo váido io lo vedo, di fronte a lo vẹdo jọjjo; nnnoem bózzo manójij non posso venire, di fronte a puó maní addomeána? puoi venir domani ? kír viéno essi vengono, di fronte a nnoen go vién miẹ́a vvodájji, non ci vengono mai a vedere.

61. L' elisione è costante tra parole congiunte pel senso.

62. L'Agnonese che parli l' italiano pronunzia strette le toniche delle parole ossitone.

\section{Consonanti. 2}

\section{Consonanti semplici.}

Esplosive.

63. Inizialj: le tenui restano intatte: kapézzo cavezza, kavallo, kukkjiéro cucchiaio; cakéura cicoria, íẹ́rro cerro; tọ́šo tosse, táuro tabula $<{ }^{*}$ tavula $<{ }^{*}$ taula $<*^{*}$ tóla; pasillo pisello, papiẹllo carta scritta. - ku - resta avanti a: kuূeáso quasi, kuándo quando; da $\varepsilon$

1 Questa norma costante di ragion fonetica non mi sembra che conforti 1' opinione del Goidanich (Misc. Asc. § I I l. c.), che cioè gl' infiniti sono accorciati sul tipo del vocativo: Totò, bęlla fẹ ecc.

2 Cfr. continuamente la Fonetica di Campobasso del D' Ovidio.

Zeitschr. $t$ rom. Phil. XXXIV. 
spesso avanti e, i: cinģo cinque (il cui $c$ del resto è già latino, ćgrko quercia. Molto di rado si nota qualche scadimento, come in wedš quasi (cfr. l' it. cen. guasi). Talora ku - perde l' elemento labiale: kattuódoco accanto a kua-, i compostî di kákks qualche, kakkáuso, kakkuwięllo qualcuno ecc.; kẹsto questo, késse cotesto, kẹllo quello, i soliti $k i$ e $k e$, accanto a kuisto, kuisso, kupiro, nei quali pare che - u- sia mantenuto da - ú finale. 1

64. Delle sonore restano: b, rafforzandosi sempre in $b b$, bbięlla bello, bbusógjjo bugia; d, doróito dietro, doláura dolore, e rafforzandosi ddojji dio, tuzźfin dozzina. Scadono: $\mathrm{b}$ per lo più in $v$, che passa in $w$ a contatto di $u:{ }^{2}$ varọilo barile, varviéro barbiere, wuóno buono, vásso basso; $\hat{\mathrm{g}}$ sempre in $h$, leggiera aspirazione sonora, hállo gallo, hastójjo gastigo, dalla fase intermedia *ga-, come pure hámmo gamba, hálto gatto; ğ in in w, wardeá guardare, wérro guerra.

a) postoniche:

Mediane.

65. Le tenui si mantengono tenacemente: leáka lago, potóika bottega, dọito dito, kuópo capo, dóico dici; cosi nella penultima dei proparossitoni: dićara, kaparo capi, jéttoko io sussulto, akuoero aghi, lgkara luoghi; -per-arkíparo ricupero; ma pǵvara, raćévara ecc. con scadimento, e son voci di lingua letteraria.

66. Delle sonore resta d, pbido piede, madoniúdo nudo; scadono o si dileguano: $\hat{\mathbf{g}}$ kjęho piego, jiuwo giogo, riuws stradetta ruga, réwoelo regola ( $w$ è sviluppo succedaneo di $u$ ); b in $v$, kánvo canapa, kućivolo di facile cottura; $\overline{\mathbf{g}} \mathrm{u}$ - perde l' elemento labiale in léngo lingua, lo conserva in sánğ $\xi_{2}$. Talora si rafforzano, specie b: sikoro sigaro, stúpoto stupido, súbbato, stábbalo letame, skupóino scobina, spárato con $\hat{\mathbf{g}}$ in $\hat{c}$ per azione del plurale sul singolare. ${ }^{3}$

b) Protoniche:

67. Le tenui restano: voćsino vicino, matóino mattina, kapézzo, kupiérkjo coverchio; $\mathbf{k}$ scade se è immediatamente prima dell' accento: pahed pagare, prohed pregare, awdinno quest' anno, fruwuó consumare, con $w$ sviluppo succedaneo di $u$.

68. Delle sonore resta d: adukkjif scorgere, kadi cadere; scadono o si dileguano: $\hat{\mathbf{g}}$ ahuisto agosto, b maravẹtl meraviglia. Notevole è il raddoppiamento di $\mathrm{d}$ in lunoddi, martoddi ecc. Finale d resta nella frase ked $e ?$ che è?.

\section{Spiranti.}

69. Iniziali. Intatti: j jonneára gennaio, jüngó giunco, joruó giurare, jénaro genero, jęnda gente; $\mathrm{v}$ vadé vedere, vẹnno vendere;

1 Cfr. Zingarelli, AG. XV $\$ 83$; De Lollis, AG. XII, 20 n.; d' Ovidio, AG. IV, I5I $\mathrm{n}$.

2 Cfr. il vastese in Rolin, l. c., 20.

Cfr. Salvioni, Rom. XXIX, 549 sg. 
f fawrinta favonio, folóitı fuliggine, feámo fame; s sdlt salire, sẹkso siccitd. Cambiano: $\mathrm{j}$ talora in $g^{\prime} g$ (il $g$ suona sempre intenso),

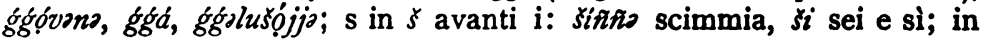
$z$ spesso: zók ${ }^{2}$ fune, zúfflo soffio, zoneálo senale; $-\mathrm{v}$ in $w$ per contatto di $u$ o di parole germaniche: wašteá guastare, wuódo passaggio, wérro verres.

70. Mediane. j resta, e postonico si raddoppia: kuarajesomo quaresima, majesstro maestro, pajaisa territorio, pejjjo peggio, frijjo friggere, léjjo leggere; viene assorbito nella penultima dei proparossitoni: freána, però wuójjata da *vǒjitu $<{ }^{*}$ vŏcitu; più che infezione labiale, in néng passa in $w$ a contatto di $u$ : drvorti divertire, kruwatto cravatta; si dilegua talvolta: niého neo, pajiuro paura, con $h$ e $j$ succedanei; rarissimo, e forse importato dal basso molisano, -ajja da -ēbam, -at, di fronte al comune -áiva. f resta: skofajé schifare; e skarafáun scarafaggio, skréufo ecc. con $\mathrm{f}$ italica, e forse pure feáfo fava, se non è per assimilazione. $\mathrm{s}+\overline{\mathrm{i}}$ passa in $\xi_{\text {: }}$ kufeáso 1 quasi; avanti -i- antivocalico: forlošójjo frenesia, malvašójjo malvasia ecc.; avanti -ēs: pajọiš paesi, Añunóiš Agnonesi, Kakkawunóiłs abitanti di Caccavone, avišš avessi, kandišš cantassi ecc.; - finale diede $*_{-i}$, che dileguandosi agì sulla tonica precedente secondo le leggi gia viste.

\section{Liquide.}

71. Iniziali o mediane restano. Finali, di monosillabi restano, altrimenti si dileguano; gli esempi sarebbero superflui. Nelle particelle si dileguano, anche se queste siano monosillabiche: $k u$ e kos con, po per, nnó non e nno, con rafforzamento consonantico.

72. In particolare è da notare la normale reduplicazione di $\mathrm{m}$ in penultima di parola sdrucciola; - $\mathrm{I}$, conforme al tipo napoletano, spesso passa in $r$, specie nell' ultima di un proparossitono: rásaro, dajgero diavolo ecc. $R u$ il, ro i, kugóiro quello, kóiro quelli son forse prodotti sotto l' azione di - $u$, -I finali, per quella stessa tendenza che nell' alatrino, sorano ecc. produce l' ammollimento di 12 ; cioe indebolito $-11-$ in $l$, fu possibile il passaggio di $*-1 \mathrm{n}$, * =li in $-r u,-r{ }^{3}$; a ogni modo il fenomeno è sporadico. 1 in $n$ núsko losco.

\section{Nessi di consonanti.}

73. j postonico complicato raddoppia la muta che lo precede, o il suono succedaneo.

dj-, ǵj-, assimilati a $\mathrm{j}$, ne seguono gli esiti (cfr. $\$ \S 69,70$ ): jürno giorno, uójjo oggi, séǵǵ sedia; cosi vj-, bj-: ajjo ho, rajjo

1 Da un * quasī, cfr. Merlo, Rev, dial. r. I, 4I3, n. 5.

2 Crr. Merlo, Sond. Zeitschr. 1. c.

Cfr. Zingarelli, AG. XV, 227. 
rabbia, kajéculo gabbia, lięggo * leviu. In parole dotte o semidotte dj- diviene doj-: dojfuro diavolo; - mediu dà miéso, *-idjare -ajé manaje maneggiare ecc.

$\mathrm{kj-}, \mathrm{kuj-}, \mathrm{pj-}$ passano in $\varepsilon$ : fácto faccio, lócto sedano, vrócto braccio, sát̂́o so, pot́táuno piccione.

tj-1 $<z$ e spesso $\ell$ : prizza pozzo, kjézza piazza, mannézza immondezza, arratoruó *adroteolare rotolare, su cui ruọ́coro rotolo, ćaloked *titillicare, skuọ́rce guscio, katćajé cacciare; dà s̀ in rašenojé ragionare, se viene da *ratjonidjare, come suppongo.

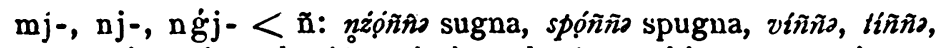
valléññ vendemmia, skañié scolorire, kañié cambiare; una riserva è da fare per la $\mathrm{I}^{2}$ del pres. indic., come si vedrà a suo luogo.

sj- $<\xi$ (non c' è raddoppiamento dopo l' accento): keáš

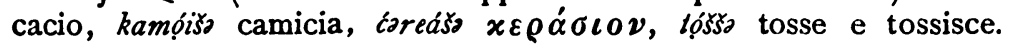

rj- postonico perde -j-: maheáro *magariu ecc., o lo rigetta abbreviando la sillaba precedente: ćgira ecc.

lj- < I: fill figlio, píll piglio, sállo salire; sporadico è jo gli illī, illis, fenomeno che connette l'agnonese ai dialetti centromeridionali (v. Merlo, l. c.).

74. kl-, tl-, pl-primari e secondari danno $k j$ : kjameá, kjęndo pianta, viẹkkjo vecchio, kkju più, kjúmmo piombo. Tra vocali $\mathrm{kl-}$ spesso dà -l-: málto maglia, vandalto, kunillt; - in una cospicua categoria di parole $\mathrm{kl}$ - secondario, e molto di rado primario, resta intatto o con o epentetico: karrúkalo arnese di legno da mettere sul basto per portar covoni, kúgkklo palle, zókklo sorcio, sklameá gridare, f. sklǵstro colostro, klućcié scricchiolare, kuklikkja ciottolo (cfr. kuókklo); - tul- al solito si riduce a ll- in spálla, ecc.; e così dul-.

ĝl- dà $I:$ lénno ghianda, lọtto glutire.

n $\hat{g} 1-<n: \phi \tilde{n} \tilde{n}$ unghia, Ań́na *angulone, ${ }^{2}$ e la forma della collina su cui giace il paese risponde bene all' etimologia.

bl- protonico dà $\hat{g} \hat{g} j$, e meno volgarmente $j$; postonico $\widehat{g} \hat{g} j$

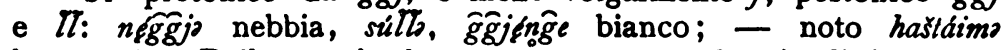
bestemmia. Delle parole lo conservano quando sia di fase neolatina, anche con o epentetico: abblaito bietola, bbubbláan babbione, subblo subbio.

$\mathrm{fl}-\langle s$ : šiumo fiume, sáimo piena, sost. mistione di flūmen e plē na. ${ }^{3}$ E conservato in zúfflo soffio, zuffled sifilare, zufflatiuro soffietto; siéno fieno è analogico su $\xi$ da $\mathrm{fl.} .5$ La persistenza in

1 Rispetto al $c$ da tj- v. Merlo, Mem. sc. Tor. s. II, LVIII, I63.

2 Cfr. De Bartholomaeis, AG. XV, 330, e Salvioni in Rend. ist. lomb. s. II, XL, I I10. La forma medievale Anglona per Agnone si trova nell' Ital. illus. del Biondo, reg. XII.

V. Salvioni, App. merid., p. 39.

4 Cfr. Ascoli, AG. X, 8.

- Cfr. Merlo, Rev. dial. rom. I, 250. 
qualche caso di bl-, fl- comincia a mostrare una tendenza che diviene legge più su. E da osservare del resto che 1 complicato, se persiste, spesso si risolve in r complicato; anzi si pud dire che, se -1 non si risolve col jotacismo, passa di regola in - $r$; e il nuovo gruppo suona chiaro, senza epentesi di $\partial$ : ćllro ragazzo, máskro maschio, simbro semola.

75. kr-, tr-, pr-, fr- restano: ákro, treávo, pruóto prato, frọnno fronda. C' è scadimento di $\mathrm{kr}-$ in $h r-, r-$, attraverso ${ }^{*} \widehat{\mathrm{g}} \mathrm{r}-$, in numerose parole: hrameá trar guai clamare, hrásso grasso, ratilto graticola.

ğr- <hr: hreána grano, hrattakeáš grattugia, hrilla grillo, hrunzeára grondaia, hrọtta grotta; - postonico: nóiro nero.

br- talvolta resta col solito suono intenso, di regola scade in vr: bbrohánde brigante, vruóda brodo, vruótara foruncolo, vrašiéro braciere, vrénna crusca, livra libbra, vritto sporco.

vr- resta: vrohọññ.

dr-protonico resta con o epentetico: dargito; postonico < tr: kuatro quadro.

76. $\hat{\mathrm{g}} \mathrm{n}$ - oltre al riflesso $\tilde{n}$, che postonico suona doppio: sláñia, nzééña poco, dassiñño sfregio, dassañed sfregiare; per *jn $<$ jon-: kajoneáto cognato, ájono agno, štajoneáto, stagnato; - talvolta $-j$ - e assorbito, cosi in láino (< lenə) legna, 1 práina pregna;

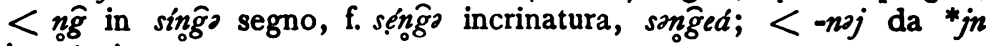
in primajo.

-ğm - < -lm-: sálma.

77. $-1+$ cons. -1 , seguito da dentale sorda, palatina o sibilante, si mantiene e rende sonora la consonante seguente, arrestando

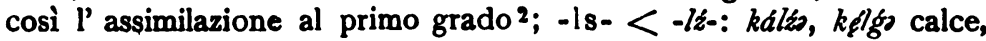
falǵáun falcione, fúldo folto, salǵtíćo salsiccia, męlís milza, múldo avv. molto, múlzo fusione, pọlǵs pulce, sáldo prato saltu, vóldatięla capogiro, prilka polso, pulkanétto paiolo, spuldróina poltrone.

$-1 \mathrm{~d}-<-l l-:$ kalla caldo, kalladro caldaia, mallitto maledetto da $*_{\text {mal-dgttu. }}$

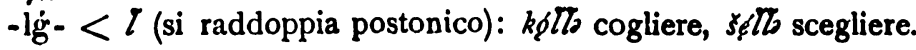

78. -r + cons, resta: bburddsły ragazzo, kurnikkjo angolo, kurtóina piccolo piazzale innanzi a fabbricati di campagna *cortina *cohortina. Scadimento di - $t$ - in -d-: mérdo io merito, mordeá meritare, ${ }^{3}$ vardá verità, sórda * sorata, spirdo ecc., sempre per dileguo di vocale intermedia.

-rs- < -rz-: vórza borsa, kğrza corso, parzáuna persona; $<$ forse $-\dot{c}^{-}-$in muićaka.

1 Cfr, il vastese in Rolin l. c. 24, e v. l' analisi del Merlo, Mem. sc. Tor. s. II, v. LVIII, p. I49 sg.

2 Cfr. Merlo, Rev. dial. rom. I, 247.

( Cfr. l' alatrino in Ceci, AG. X, I69. 
79. $-s,-x+$ eons. $C^{\prime}$ è $s$ solo innanzi a sonore; è $\xi$ avanti a dentale: stallajjo stallaggio, stzzzoje piovigginare, stuted smorzare, šdumodí sgonfiare, šduósso a schiena.

skj- primario o secondario da $\zeta k j,{ }^{1}$ e $\xi$ resta pure se $k j$ passa

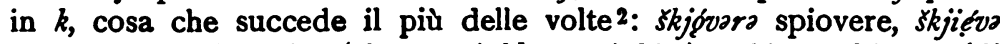
vento di Schiavonia (sla- $<{ }^{*}$ skla- $<*^{*} k j a-$ ), skitto schietto (di panno), sksppo schiappa, skuppẹtto schioppo, skaffáun schiaffo, škameá gramare, piêsks macigno e Piešs paese *peslu, 3 škiumo, maškatiuro

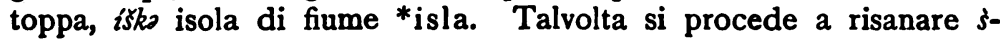
in $s=:$ skttto solo avv. svolazzo.

sv - < sbb-: sbbaleá scoprire, sbbrohuñiẹto svergognato, śbbalákkja

$\mathrm{x}$ - intervocalico dà -ss-: assukuó asciugare, kósso coscia, ssuọ́mo sciame; in altra posizione segue sempre le sorti di s. Nota sénno ala, mistione di axilla e ascendo.

Il solito (v. Misc. Asc. 8o) kášš da capsa.

8o. $-n,-m+$ cons. - Le nasali seguite da $b, v, f$, passando per *-mb-, danno -mm-, che iniziale spesso si scempia: hammeáca bombagia, mmatiuto imbattuto, ammokkuó *imbuccare, ammotojé invitare, ammálto, nel caso che $<^{*}$ in-facto, kummóito convito, molikuoero ombelico, 4 massseáto imbasciata servizio; - del resto - $\mathrm{nf}$ di regola dà -mb-5: mbónn o bagnare, ${ }^{6}$ mbátća in faccia. In bambóina specie di uva, da *bon-vinu, l' assimilazione s' è arrestata al primo grado, per la recente composizione verbale.

$\mathrm{n}, \mathrm{m}$ : rendono sonora l' esplosiva muta mbénno appendere, $a$ Il' andrasditt all' improvviso *in-trans-acta, nnoen ga viéno, angaduro ancora, frọnda fronte, jọndo giunta, nógiénta incenso, nğóino uncino, ñ̂jjané salire *in-planare, nĝjestro empiastro; - in jünǵs giunco, péngo tegola, il -g- è tratto dal plurale.

-nd- <nn: banno bando, mazzaśǵnno mazza-fionda; di rado tuttavia il gruppo resta: kuándo quando, addóndo dove ecc.; oscillamento tra $-n n-e-n d-8$ che è segno di un' assimilazione del gruppo non molto remota. ${ }^{9}$

$-n \dot{g}$ - è regolarmente assimilato a $-n j-$, onde $n$ : mantié man-

1 Cfr. Merlo, Rev. dial. rom. I, $258 \mathrm{sg}$.

Cfr. Zingarelli, AG. XV, 93.

3 V. Zingarelli 1. c., e De Bartholomaeis, AG. XV, 351 .

4 Cfr. Salvioni, App. merid., 32.

- Cfr. Zingarelli l. c.; Merlo, Rev. dial. rom. I, 255.

- Pel significato cfr. De Bartholomaeis, AG. XVI, 67.

7 Cfr. Salvioni, Rom. XXIX, 551 .

8 Vedi Meyer-Lübke, R. G. I, 4I9; per lo stesso fenomeno a Scanno v. Merlo, Rev. dial. rom. I, 417 .

- Il De Lollis (Misc. Asc. 1. c. § 17 ) vorrebbe vedere *-nd- in kiññ e ñरe ñra come, diffusi variamente in tutto l'Abruzzo, facendoli derivare da quanta; ma non pare accettabile l' ipotesi dell'A., sia perchè mancano altri esempi di $n t<^{*} n d<\tilde{n}$, sia perché ašsệññ scendere, che potrebbe confortare questa opinione ha bisogno esso stesso di spiegazione. 
giare, tolóino gingiva ${ }^{*}$ gingilina ${ }^{1}$; fütito è sul plarale, fungo -imp- <n: éñĩa empiere.

- $n$ - seguito da sibilante primaria o secondaria la rende sonora, se esso non cade: lanźuọra, pánźa pancia, kapasojé calpestare mistione di ${ }^{*}$ pinsiare ${ }^{2}$ e calpestare, masélla madia *mensilla. Nota il solito ssallúzza.

\section{Accidenti generali.}

81. Aferesi della prima sillaba scambiata per articolo in sáñña lasagne.

Protesi frequente di $j$-: jérvo erba, jéttoko etica, jérto erta; frequentissima di $h$-, leggiera aspirazione; hastáima, họ́lpo volpe, húmato tuono; e quest' aspirazione impedisce l' elisione. Prostesi di $h$ - avanti a $r$, per falsa ricostituzione verbale, su $h r-\mathrm{da} \hat{\mathrm{g}} \mathrm{r}$ : hróina reni, hramoliéro ecc. ${ }^{3}$; - di $l$ per concrezione di articolo: léndro edera, léska fetta, lọ́ćs accio; di $n$ per concrezione di in: naspatiura aspo, nĝlóstro chiostro, mbriẹka ubbriaco; di ad: addummədéuro gonfiore.

La sincope di $r$ dopo dentale è rara: másto accanto a mástro.

Epentesi di $r$ non raramente: spérkjo specchio janešstro ginestra, şgrizzo schizzo, fruššllla fiscella; - di $b$ tra $\mathrm{m}$ e liquida: kámbro camera, vémbro vomero, túmbro tumolo (misura di capacità), nsiẹmbra *insếmulŭ insieme, simbra semola; di $v$ in davéndro, 4 se non viene direttamente da *de-ab-intro, come inclino a credere.

82. Assimilazione nel grado: dọddo dote, duddello, dudddzza; nell' organo: manójij venire, mantuto venuto e composti, frifia giungi ecc.

Dissimilazione (oltre che nei comuni kanókkjo ecc.) di $1 \ldots 11,1 \ldots \mathrm{r}$ in $n \ldots l:$ éndro altro, kundięlb coltello, se non risale direttamente a cuntellus; - di $\mathbf{r} \ldots \mathrm{r}$ in $l \ldots r$ or ...l: listro arista, con epentesi di $r$ che ha prodotto la dissimilazione, ${ }^{6}$ rasuólo rasoio; - in léndra rondine da $*_{\text {rénnena, con } *_{n n} \ldots n<n n \ldots . . .}$,

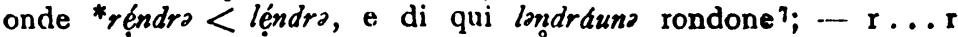
in $d \ldots r$ pródoro, il cui $-d$ - resta in tutta la coniugazione; da un

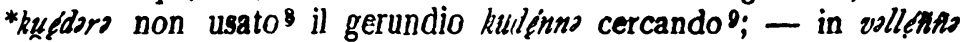
vendemmia è da vedere una dissimilazione di un anteriore vonnénñ, che resta tuttavia nel napol., pugliese ecc.; - $11 \ldots .1$ in $n n . . . r$ in pinnara pillola; $-\mathrm{m} \ldots \mathrm{mm}$ in $v \ldots \mathrm{mm}$ in vammọino levatrice.

1 V. Salvioni, App. mer., p. 20.

V. Ascoli, AG. XVI, I8I.

Cfr. Salvioni, Rend. ist. lom., s. II, XLI, 890.

- Cfr. Salvioni, AG. XVI, 68.

V. Ascoli, AG. XIII, 284 n.

- Cfr. Salvioni, App. mer., p. 6.

I Cfr. ancora Salvioni 1. c.

B Cfr. De Bartholomaeis, AG. XV, 354 .

- Cír. pure Salvioni 1. c. 
83. Metatesi comune nelle formole cons. + voc. + cons. $t r$ e cons. + voc. $+\mathrm{r}+$ cons.: fróivo febbre, bbrills burla, vritajo vetro, vrohọtito; in s. fúprcovo forbici c' è metatesi reciproca ${ }^{1}$; - spesso pure per -1-: abblaito bietola, kjuóppo pioppo.

Un filone inesauribile è nei composti col prefisso re-, onde *er-, ar-, filone comune a tanta parte dei dialetti centrali e settentrionali 2: arpọnno riporre, arsaneá risanare, arkjọino ecc. Se ar- si trova innanzi a consonante alla quale non si appoggi bene, passa in arro: arraspọ́nno rispondere, arrošš riuscire, arrastrẹñño riunire.

84. Le doppie in penultima di proparossitoni tendono a scadere nelle semplici: bbonísomo abbastanza, másəmo al più, mámoto tua madre, kuninaća quindici, uinás undici, da *kuinnaća *innaćs.

85. Raddoppiamento. A non tener conto dei casi in cui sia dovuto ad assimilazioni di consonanti diverse, ad azione di prefissi o a dissimilazione ${ }^{3}$; lasciando da parte $g$ e $b$ che intervocalici han sempre suono intenso; raddoppiano la consonante iniziale $\mathrm{i}$ monosillabi: hkju più, nno non, rre re,4 nne nec, lla là kkua qua; - la consonante successiva alla prima sillaba le parole accentate sulla terza, e talvolta anche quelle accentate sulla seconda: cammanóiro camino, pammadéuro pomodoro, vaćcóila bacile, dossiñño. -t- per altro resta scempio: matonánda mattiniero, skatonatiuro pettine rado; restano pure scempie alcune, per analogia di parole dello stesso radicale: cekalinino miope su takeáto ecc.

Estesissima è la serie delle parole che raddoppiano l' iniziale per supposto prefisso: abbaded badare, abblaito bietola ecc. - Rrobbo roba e mmérdo rientrano in questa categoria da $* l '$ arróbbo $<$ la rrobba, *l' ummérdo < la mm\&rdo, col supposto prefisso $a-$, staccato dall' articolo.

86. Le particelle fornite di virtù reduplicativa sono: \& et, nne nec, ng no, kkju più, ke, a prepos. e prefisso, pa per, koe con, se sum e sunt, $\&$ est, $\zeta_{i} i$ sei sii, so congiunz.; e ancora i pronomi kakks qualche, grins ogni, to come, che sono invece di *kdkk' $\&$, 'gnn' $e$, * $f^{3} e^{5} ; \mathrm{gl}^{\prime}$ imperativi $s t a, f a, v a$, di, che raddoppiano le sole particelle pronominali.

Il raddoppiamento avviene di regola duplicando quella che è la consonante iniziale; però $h$ - passa in $\widetilde{g} g_{-}$, meno spesso resta; $j-$ passa in $\widetilde{g} g j-$, nel ceto più civile $j j-; v$ - normalmente in $v 0$-, in composizione però in bb-: abbálla giù, abbọkka ecc.; $w$ passa in $\widetilde{g} \underline{\underline{x}}$-.

1 Cfr. Salvioni, Ap. mer., p. 3 I.

2 Cfr. Meyer-Lüble, R. Gr. I, 29 I sg.

- V. D' Ovidio, Rom. VI. Ter., 123.

4 Cfr. D' Oridio, AG. IV, I72 ; Zingarelli, AG. XV, IOI ; Sarini, Dial.

$s$ Vedi Zingarelli, 1. c. 
87. I mutamenti che avvengono nelle consonanti di una parola, succedono pure tra il suono finale della parola precedente e quello iniziale della successiva: così nu wállo il gallo, come ríuwa stradetta; nn ǵo párla, come nǵǵnna; e cosi noen źgerrr non serra, bon ni< $<$ bon di, ecc.

\section{La flessione.}

\section{Declinazione.}

\section{Nome.}

88. Nominativi sono: mólla, séura sorella, látro ladro. Bello esempio di continuazione flessiva è in s. séura e p. suriura, oltre che nel comune éuma s. e uómmana p.

89. Maschili sono: lébbra lepre, cémoća cimice, pọlǵa pulce; femminili: kaneála, kumbọino il confine, pammadéura pomodoro, reáto aratro da $* l$ areáto $<l a$ reáta, zaffareáno, panarọića patereccio, läwaro lauro, forse per la- supposto articolo, bbakkaleán baccalà.

9o. Il vocativo non riduce la parola all' accento; solo rende men chiare le sillabe postoniche. Sui residui degli altri casi non c' è cosa degna di nota particolare.

91. La declinazione succede nella tonica, sotto l' azione delle vocali finali, in conformita delle leggi fonetiche. 1 Movendo perciò dalle finali originarie, omai ridotte a -2 , salvo le riserve fatte al $\S 52$, si hanno cinque classi:

$$
\begin{array}{clcccc}
\multicolumn{2}{c}{\text { I. classe }} & \text { sing. }-a & \text { plur. }-e \\
\text { II. } & n & n & -u & n & -i \\
\text { III. } & n & n & -e & n & -i \\
\text { IV. } & n & n & -u & n & -a \\
\text { V. } & n & n & -u & n & -o r a
\end{array}
$$

92. I. classe. $\dot{E}$ indeclinabile, giusta le leggi fonetiche. SulI' analogia dei femminili della III classe hanno metafonesi al plurale?: s. fósso, p. fuissa le fosse; s. raštóćća, p. rostucićć le ristoppie; s.

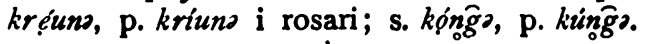

93. II classe. $\dot{E}$ indeclinabile, fuorchè se la tonica sia $-a ́$ (cfr. $\$ \S 4$ e 8): s. kualredra, p. kualriéro ragazzi; s. gjjona, p. éjono agni; s. lóćć, p. léććs sedani. È fatto sul plurale per differenziarsi qualche singolare: dal p. majistro maestri, il s. majestrtro; dal p. zinģoro, il s. zéngaror zingaro; dal p. pulgóoino, il s. pulgáina pulcino, che del resto coesiste accanto al regolare pulgóino; dal p. spóino spini, il s. spáino oltre al regolare spóino; dal p. spíuso sposi, il s. spéuso; - sull' analogia dei temi in -ọ́-, è il p. tosiuro dal s. tosẹuro tesoro. Il s. miéno mano è tratto dal $\mathrm{p}^{3}$

1 Cfr. Meyer-Lübke, R. G. II, 62 sg.

2 Cfr. Salvioni, Rev. dial. rom. I, 103 sg., per questo $\$$ e pei seguenti.

- Cfr. il reatino in Campanelli, 38; e cfr. Bianchi, AG. XIII, 198; e Merlo, Mem. sc. Tor., s. II, LVIII, 413, n. 4, e Rev. dial. I, 199 n. 
Alcuni nomi colla tonica -ŏ- hanno il p. foggiato su quello dei nomi in -á-; così i p. karéfono garofani, lémmoro gomitoli, vémbro vomeri, e simili, dal s. karøfono ecc. - L' azione analogica si spiega col $\S 8$, perchè facendo il p. fjono dal s. gjono < al più civile ájono, si rese possibile un p. kareffono dal s. karófono. $\mathrm{E}$ l' analogia è stata così efficace che al p. Témmaro risponde, oltre al volgare lómmoro, un più civile támmoro; come al volgare ǵcons risponde il più civile ácona (cfr. §6). Sul p. vémbra vomero è poi rifatto un s. f. vémbro. Nota il p. domuónaja dal s. s. d. damónəjo demonio ${ }^{1}$; p. tézwaro tori, dal s. táwaro.

94. III classe. È declinabile, secondo le leggi fonetiche; quindi non è declinabile per $-\hat{f}-,-\bar{u}-$; per questa ultima categoria ci sono dei singolari dissimilati dai plurali: s. lémoto di fronte al p. limota; s. cémó́c dal p. ć́maća ${ }^{2}$; s. pọ́lgó dal p. púlǵo; s. sọvara dal p. suiwaro. Il s. níuća noce è attratto dal p., per la prevalenza di questo su quello.3

95. IV classe. 亡̀ abbastanza largamente rappresentata. Per -á-: áćono acini dal s. géons, vráćco braccia dal s. vróćco; - per -ế-: carvélllo cervella dal s. corviẹ́llo, p. padaméndo dal s. padomiénda; per -é-: p. karézzo dal s. karizzo carezza, dáito da dóito dito, pédoto peti da pídoto ecc. Degno di nota è il plurale delle frutta che conserva il latino -a: prézzzos pesche dal s. priẹzzəka, páira pere dal s. pọiro, máila da móila, sórvo da suọ́rvo ecc.; di fronte al plurale degli alberi: móila o álbaro do móila meli ecc. - Sono tratti dal plurale, i seguenti f. s.: vosdicto, bisaccia, hréññ fascio di spighe, p'́kuoera pecora, cẹllt ciglio, hraméñio gramigna, fólto foglia, prokkéuka percoca. Tratto pur esso dal p., tuttavia di genere maschile, è lệ́ano *ilicinu.

96. V classe. È largamente provveduta dalla II; anzi sono ben pochi i nomi di cosa che non l' abbiano o non la preferiscano all' altra, che del resto coesiste frequentemente: káparo capi di filo ecc., prdior prati, pajęttoro piatti, akkuoer aghi da edks, che al s. gli Agnonesi trattano da f., illusi forse da l' aks, facendolo < *la dko; kupérkjaro coperchi, vérzoro smorfie, értara orti, fóssoro i fossi da fuósso, lọporo i lupi, órsoro orsi ecc.

I nomi in -iuro da ộriu hanno al p. -órro < ${ }^{*} \not \dot{r}(0) r o:$ pošsatórro orinali, toratórro tiretti, putatórro potatoi. Quelli considerati al § 39, hanno -ó-: jongkkjora ginocchia, kjópporo pioppi. Quelli con - $\hat{\mathfrak{f}-,}-\hat{\mathrm{u}}-\mathrm{h}$ hanno un $\mathrm{p}$. analogico sui nomi in -é-, -ọ-: marétoro mariti, varẹllor barili, nẹdoro nidi, féloro fili, kušsénoro cuscini, mandéloro tovaglie, kavọtoro buchi, fósoro fusi. - S. f. tratto dal p. è

1 Cfr. il campobassano in D' Ovidio, 1. c., 159.

2 Cfr. Zingarelli, l. c., 86; Salvioni, Rom. XXIX, 553-4, e di nuovo nella Rev. dial. rom. I, I03 sg.

8 Come in testi dell' alta Italia; v. Krit. Jahr. IX, p. I², 97.

- Cfr. De Bartholomaeis, AG. XV, 346, e Meyer-Lübke, Nltlog., 7 sg. 
fikuoero fico, s. m. è s'snğuoero fianco; c' è inoltre kdsoro p. case dal s. keáso della I classe.

\section{Aggettivo.}

97. Il passaggio della II classe alla I non è regolare ${ }^{1}$; cito: m. múlla, f. mólla; ahrięsto m. uva che resta immatura, ahréste f. uva non ancora matura; kućivola m., f. kućévola di facile cottura; m. stípa, f. Stérpo sterile; majiura primogenito, che del resto avrà una ragione propria, perchè si trova un po' dappertutto; virdo m., f. vérdo; sottiémbra, nuwiẹmbra, daciémbro.

\section{Comparazione.}

98. Comparativi organici nell' uso sono: péjjo pějor, męllo melior, pajéuro con significato intensivo nella frase kéšt $e p\left\langle e j j j^{2}, e\right.$ kést' aldr' e pajéura questo è male e quest' altro è peggio; raro è móina meno minor, 2 il s. c. majiura con significato sostantivale di primogenito, e, in composizione con si- $<$ thiu, si-majiuro avo.

Forme di superlativo organico sono: bbonisamo con significato avverbiale di assai, másımə, minəmə; raro prósəmo s. d.

\section{Numerali.}

99. Sono: iuns m. e f., diuws tonico, ed atono du, tre, kudttro,

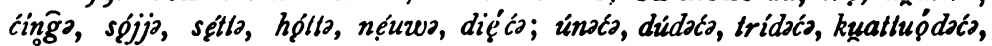

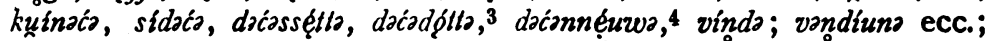
tręndo, kuarándo ecc.; ciệndo, millo, du móilo o du miloja.

Il sistema dodicesimale e quello vigesimale sono usitatissimi, specie, com' è naturale, per designare oggetti e lavori campestri.

\section{Pronomi personali.}

100. Le forme toniche volgari sono: jọjjo io, tiuzwa tu, niuwo e viuzes; cui rispondono i più civili $j i j o, t u, n u, v u ;-m e, t \ell$, e coll' epitesi méjo téjc. - Le atone sono: $j i, t u, n u, v u$, to ci, vo vi, mo mihi, e te ti, sos; la, lo; ro e lo maschili, dei quali il primo si riferisce a persona o cosa determinata, il secondo a quantità o cosa indeterminata; quindi di uomo $r o$ vdido, di pane lo vdido. Col Merlo (Sond. Zeitschr. l, c.) è da vedere in ro il continuatore di ill $u$,

1 Il criterio che deve guidare il glottologo nella ricognizione delle classi degli aggettivi, in questi dialetti a flessione interna, è lo studio della tonica. Perciò non è esatto quanto il de Lollis afferma (Misc. Asc. 275, § 4), che nell' Abruzzo sia costante ii passaggio degli aggettivi dalla seconda classe alla prima. Il vero $d$ invece che $i$ f. seguiti dal sost. si fanno uscire in $-a$; ma questo non el passaggio di classe, in dialetti a flessione interna; ma e fenomeno in gran parte fonetico, in parte pure analogico, e si estende non solo agli aggettivi, ma a tutte le altre parole, come lo stesso autore egregiamente dimostra (cfr. $\$ 53$ ).

2 Cfr. Merlo, Sond. Zeitschr. XXX, 444 sg.

8 V. Zeitschr. XXIII, 518-9.

4 Il raddoppiamento -ss-, -nn-, et dovuto alla cong. e.

- Per l' etimo vedi D' Ovidio, AG. IX, 66, n. 2. 
in lo di *illod *illoc, foggiati su hoc quod.1 Il p. però è sempre $r o<$ illi. $\dot{\mathrm{E}} j$ il continuatore di illi illis; co con significato di gli non è nell' agnonese. In enclisi e proclisi sempre mola, talo, mono, colo, volo, jolo; no enclitico mantiene $n n$, e richiama l' accento sulla particella precedente: jamoćinno, vattínno ecc.

Pronomi di $3^{2}$ pers. sono i dimostrativi. Manca il pronomen reverentiae.2

\section{Possessivi.}

101. Son sempre posposti al nome cui si accompagnano. Forme toniche sono: m. s. e p. mójja, f. s. e p. májja, e cosi tójjo e tájja, sójjo e sájja; m. nuóstrtr, f. nóstro; e così vuóstro e vóstro; léura loro. Mọjjo e il più civile míjo sono, come il toscano mio, da ${ }^{*}$ miu $<{ }^{*} \operatorname{mieu}^{3} ;$ májjo e il più civile $m e j j$ invece sono da ${ }^{*}$ mea, fase posteriore di $*$ miea. Il possessivo della $2^{2}$ e $3^{2}$ persona è fatto su quello della $I^{2}$. Forme atone sono $-m a-t a$, usati coi nomi di parentela: mámota tua madre, sórdo tua sorella.

\section{Dimostrativi.}

102. $\mathrm{Da} *$ 'llu, $-\mathrm{a}, *-o c$, in funzione di articolo $r u$ e lo m. s., ro m. p., la f. s., la f. p. Per l' uso di ru e la vedi il § roo: dámmo lo peáro, dammi il pane; vído ru pulǵáino vedi il pulcino; fea lo kjuvalokillo pioviggina ${ }^{4}$; innanzi a vocale però sempre l' s. e p.

$\mathrm{Da} *_{\mathrm{ipsu}},-\mathrm{a}, *_{-} \mathrm{oc}$, forme toniche m. hissa, f. hésso, con significato personale e una sfumatura di $\mathrm{ipse}^{5}$; atone ssu e sso m. s., ssa f. s., ssa p. m. e f., cotesto, in cui il dimostrativo s' attenua e vanisce a poco a poco fino all' articolo. 6

$\mathrm{Da}$ *'stu, -a, *oc, le proclitiche $s t u$ e $s t a$ m. s., sta f. s., $\zeta t o$ p. m. e f. Per la differenza tra $s t u$ e $s t o$, ssu e sso vale quanto si è detto al $\S 100$.

$\mathrm{Da}{ }^{*}$ 'kku-istu, *-a, *-oc, kuisto e kẹ́sto m. s., kẹ́sto f. s. e p., kiśto m. p., questo.?

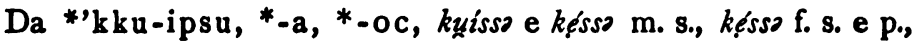
kisso m. p., cotesto.

1 Ctr. Ascoli, AG. XIII, 294, e Meyer-Lübke II, 122.

2 Cfr. il reatino in Campanelli, 126.

3 Vedi D' Ovidio, AG. IX, 45 sg.

- Cfr. Campanelli, 128.

- Cfr. Ascoli, AG. XV, 314.

- Cfr. Ascoli, 1. c., e Parodi, AG. XIII, 304.

T Pel m. kẹsts c' è l' ipotesi del Goidanich (Misc. Asc., 401 sg.) che esso rispecchi il p. n. latino; opinione a cui giunge anche il de Lollis (op. c., 275, § 12), sebbene avesse prima (AG. XII, 20) postulato un *ecu-illod pel m. kêllo; c' è poi l' ipotesi del D' Ovidio, per cui kẹśto sarebbe s. f. coll' ellissi di cosa. Ma da quanto si e visto risulta chiaro che kẹśto, oltre a stare insieme con kẹllo e kẹsso, è da mettere pure insieme coi m. s. lo, sso, što; e la spiegazione ha da servire per tutti. Percio sono interamente col Merlo nel riconoscere la necessita di postulare un *istoc, *illoc, *ipsoc. 
$\mathrm{Da}{ }^{*} k \mathrm{ku}$-illu, *-a, *-oc, kugiro e kẹllo m. s., kẹllo f. s. e p., kóira m. p., quello.

$\mathrm{Da}{ }^{*}$ 'st-ipse, ${ }^{*}-\mathrm{a}$, m. e f. stẹsso.

Altri pronomi.

I03. $k i$ è interrogativo di persona e di cosa; kújjo, da cuju, $-a$, vale chi?

104. 'L' indefinito iuna, in funzione di articolo diventa $n u$ no m., $n a$ f.; e la differenza che passa tra $n u$ e no è quella stessa che passa tra ru e ro. kuwiẹlla nessuno *qu ovelli, kubbęllo niente *quovelle; quindi kakkuwiélla qualcuno, unğuwiẹlls ognuno *omn-quo-; kakkodíuna, kakkáuso qualcosa.

L' indefinito italiano $s i$ è reso in agnonese con $\gamma_{3}$, di fronte al so personale: ša váida si vede, ma so la máññ se lo mangia; $y_{\partial}$ leávo koe lla sapéuna si lava col sapone, ma hisso sa lcávo la fáćco egli si lava la faccia.

\section{Coniugazione.}

105. I. Ad -áre rispondono tre classi di verbi:

a) vocale tematica $-d-:$ abboled, kanded; b) vocale tematica -ó-: allokkuó, mosoruó; c) vocale tematica -q́-: skuntié, nzeñié.

II. -ếre, Lĕre si riducono ad una classe, fuorchè negi' infiniti:

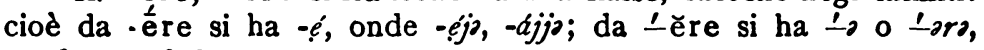
conforme al $\S 59$.

III. - fre conserva $-i$, onde il volgare -ojjj da $\hat{\imath} j$ : song $d i$ ecc.

106. Rispetto al contenuto delle varie coniugazioni, noto il passaggio di -ére in Lēre in ẹ́ña empire, móññ mungere, móvoro; - Lerre in ére in sapẹ; -ếre in fre in $\pi i$, accanto al s. c. ęrno, armani rimanere; - - ĕre in -fre pardt perdere, fuji fuggire, kadí cadere, kusí cucire; - - ĕre in -áre tromed; - -fre in -ð re pródoro, lótto inghiottire; -áre in -ére, per coincidenza fortuita, nella cospicua categoria dei verbi in -ajé, provenienti da -igare,

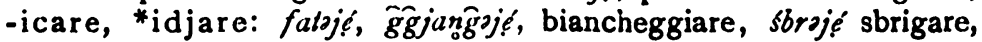
umotoje tonare ecc. (v. $\S 3$ )。

\section{Desinenze personali.}

107. $I^{2}$ pers. - Originario - $0<$ agnonese -0 nell' ind. pr.; - origin. $-\overline{1}<$ agn. -2 , con metafonesi alla tonica nel perf. ind.; orig. $-\mathrm{e}<$ agn. -2 nell' impft. cong.; - orig. - a < agn. - -2 e $-a$ (secondo il §53) nell' impft. ind. e condiz. pr.

$2^{2}$ pers. - Orig. $-\overline{1}<$ agn. -2 , con metafonesi alla tonica, nei vari tempi e modi. Orig. $-\mathrm{a}<-\rightarrow$ e $-a$ nell' imperativo.

$3^{2}$ pers. - Orig. $-a<$ agn. -2 e $-a$ nel pres. ind. I, cong. pr. II e III, impft. ind. sempre; - orig. -e < agn. $\rightarrow$ nell' ind. pr. II, WI, perft. indic., imperft. cong., pr. cong. I. 
$4^{a}$ pers. - Orig. -ámu $<$ agn. -edmo nell' ind. pr. I a, imperft. ind.; < agn. -uómo nell' ind. pr. I b; < agn. -iémo nell' ind. pr. I c; orig. èm $\mathrm{u}<$ agn. -dimo fatto analogicamente su -ditol nel pr. ind. II, quindi senza metafonesi; - orig. -im u < agn. fimo nel pr. ind. III; - orig. -ssẹmu < agn. -sş́ima, con metafonesi, nell' impft. cong. Il perf. indic. ha $-\dot{g} m m$, esteso a tutte le coniugazioni.

$5^{2}$ pers. - Orig. -áte < agn. -eato nel pr. ind. Ia, imperf. ind.; < agn. -uọ́to ind. pr. Ib; < -iéto ind. pr. Ic; - orig. éte < agn. -dito ind. pr. II; - orig. - fte $<$ agn. -óito ind. pr. III; - orig. -ssẹte < agn. -ssóita, analogico su -ssó́imo. Al perf. ind. c' è -ǵsto per tutte le coniugazioni.

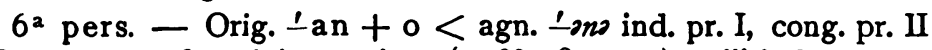
e III, con metafonesi incompleta (v. \$\$ 28 e 45) nell' ind. pr.; orig. - $\mathrm{un}+\mathrm{o}<$ agn. '-ano, con metafonesi per $-\mathrm{u}-$, ind. pr. II e III; - orig. \pm en + o < agn. - ano nel cong. pr. I; - orig. '-ban + 0 $<$ agn. -vano impft. ind. - orig. *-ĕ́run $+0<$ agn. -iérno nel

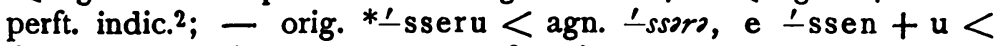
'ssona nell' impft. cong., con metafonesi.

Presente indicativo.

108. Modelli:

I a. kándo, kiẹndo, kándo, kandeámo, kandeálo, kándono, canto.

Ib. allikko $1^{2} 2^{2} \mathrm{e} 3^{2}$, allokkuọmo, allokkuọto, allíkkano, grido.

Ic. máñ̃a, miẹññ, máñna, mañiẹmo, mañiẹta, máñ̃ana, mangio.

II. váida, vọido, váido, vodáimo, vadáito, vídano, vedo.

III. párto, piérto, párta, partóimo, partọito, piértono, parto.

Ioga) I verbi in - $l^{\prime} g$ - hanno tre esiti alla $I^{2}$ p.: o -lg-, o -IT-, sull' analogia di tutta la coniugazione, o -nğ-. Più schiettamente volgare è $-n \hat{g}_{-}$, meno $-l g-$-, abbastanza diffuso - $l l-$; del resto

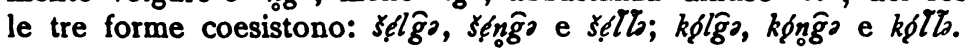

b) I verbi in - $n \dot{y}$ - hanno - $n$ - per tutta la coniug.; per la $I^{2} \mathrm{p}$., oltre all' analogica in $-\pi \hat{n}-$, più usata è $-n \hat{g}-:$ téngo e tẹññ,

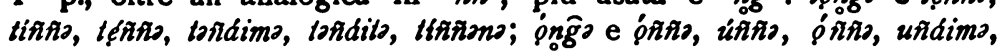
unáito, uintions.

c) Per l' influsso della vocale derivativa noto: faćo fo, stićo so, ájj ho, pózzo *potjo, wuóllb voglio, sált salgo. Accanto a

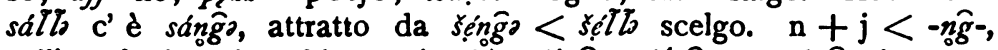
sull' analogia dei verbi notati a b): tiệnğe, viẹng

1 Cfr. Goidanich, Misc. Asc. p. 401, § 10. Vedi pure altri dialetti in Parodi AG. XIII, § 5; Campanelli 56; Savini 68; D' Ovidio AG. IV, 184; Merlo, Rev. dial. r. I, 4I5, ecc. Il Merlo (n Gli italiani amano, dicono ecc. ${ }^{4}$ ) ammette che $n^{\text {al }}$ class. - $m$ ŭs di prima plurale fosse venuto sostituendosi nel volgar latino d'Italia, e forse non solo d'Italia, un ipotetico * $m o s^{\prime}$ “. Però è da notare che $i$ dialetti napoletani mostrano invece l' azione di - $\vec{u}$; quindi, se mai, il * mos, sarebbe da attribuire ad una zona solamente. Confesso però che mi sembra più accettabile la surriferita ipotesi del Goidanich.

9 Cr. Salvioni, Stud. fil. rom. VII, 208; Merlo in Rev. dial. r. I, 4 I 4. 
arpọnḡe, accanto a arpọnna. Degni di osservazione sono assẹnĝ̀ scendo e arrénĝ, rendo (cfr. l' umbro descengo) fatti su tẹnģa; e

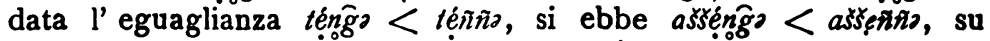
cui poi l' intera coniugazione; non così però di arrẹng. quale $\mathrm{l}^{\prime}$ analogia si è arrestata alla $\mathrm{I}^{2}$ per.

d) Gli altri verbi in gutturale mantengono la faucale alla $1^{2}$ p., mentre prendono la palatale alla $6^{2}$, per azione di $-2-$ di -ənə, conforme al nap., bar., ecc.: kẹ́ukə, ma kuọ́conə; artórka, ma artuọrcóñ; dọiks, ma dićona. Così pei verbi in -scere: krẹska e kriššsna, arnáska e arniẹ̌šana; sui quali ęska esco e tọska tossisco.

e) Verbi incoativi ci sono, e generalmente gli stessi della lingua letteraria; la flessione è limitata come d' ordinario: forniska, farnišsa, farnišš, farnọima, farnọita, forniššna, finisco.

I 10. Osservazione speciale meritano i seguenti verbi:

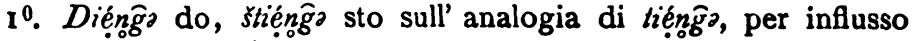
della $2^{2}$ s. e $3^{2}$ p. p. ${ }^{1}$

$2^{0}$. Accanto a vdjjo vo, da *vadjo, c' è váltb.

30. Dié, stiẹ, fié, viẹe, siẹ, jẹe, puó, wuớ, regolarmente da dai, stai, fai, vai, sai, ai, prooi, vuoi ${ }^{2}$; le $3^{\mathrm{e}}$ per. deá, steá, fed, ved, sed, eá, pẹu, vẹeu; le $6^{\mathrm{e}}$ diẹ́na, stiẹna, fiẹna, viẹ́no, siẹno, jệno, puớno, wuọ́no sono tratte dalla $2^{\mathrm{a}}$ p., come piẹ́rtono partono pare foggiato su piẹrrto ecc.

40. Andare non c' è; ire si alterna con vádere nella flessione: vajjo, viẹ, veá, jẹmo, jẹto, viẹnn; việnga, viẹ, vojjjo, monọimo, monóito,

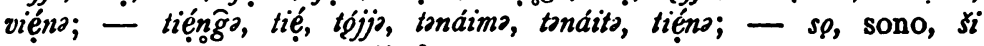
tratto da sīs, e, sáimə, saito, ${ }^{3}$ sẹ.

\section{Congiuntivo presente.}

Ix 1. Modelli: Ia, b, c, $3^{2}$ kándo, $6^{2}$ kdndona; II $3^{2}$ vdida, 6a

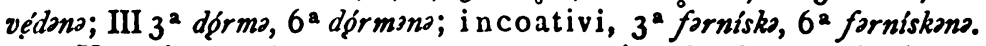

Vere forme di cong. pr. sono, come si vede, la $3^{2}$ e la $6^{2} \mathrm{p}$.; le altre sono sostituite dalle corrispondenti del cong. imperft. Valgono poi per esso tutte le osservazioni fatte al $\S 109 a$ ), b), c), d) e) per la $\mathrm{I}^{2}$ dell' ind.; perciò sénğa sénğgno scelga, pózza pózzano possa; vẹnğ vẹnğono vinca; kẹuk kipkiane cuocia; fornisko forniskano finisca.

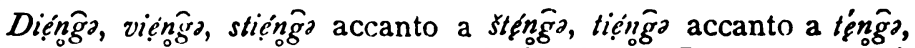
sono interamente foggiati sulla $\mathrm{I}^{\mathrm{a}}$ dell' ind. - La $2^{\mathrm{a}}$ puózza $\mathrm{e}$ dalla $3^{2}$ pózzo, come la $2^{2}$ puó dell' ind. sta a po $3^{2}$ per.

\section{Imperativo.}

I1 2. Modello: I kándo $2^{\text {a }}$ per. - La II e la III non hanno forme particolari, e prendono la $2^{2}$ dall' ind.; tutte le coniugazioni

IIl teramano, chiudendo il dittongo, ha dinĝ̀, štinĝe (v. pure Salvioni, St. fil. rom. VII, 202; Pieri, AG. XVI, I64).

Il teramano, chiudendo il dittongo, ha di, fi, štil ecc.

II Meyer-Lübke, II, 252, postula un *sętis. 
poi hanno la $5^{2}$ dall ind., la $3^{2}$ e $6^{2}$ dal cong. Conservati sono $d i, f a$; c' è pure il comune va. $\mathrm{Dal}$ cong. è tratto anche puógzo $2^{2}$.

Rispetto all' uso nota le frasi: váll' a thuólta, valt' a dduórmo, váttal' a mmáñno, va ssẹ́uno, con $a<$ ac.1

\section{Indicativo imperfetto.}

II 3. Modelli: Ia. kandedva, kandọivo, kandeávo, kandaveámo, -avedto, -ávono.

Ib. allokkuọ́vo gridavo, allokkọivo, -uộo, allakkaveámo, -aveáto, allakkgoono. ñévona.

Ic. mañiệ́ mangiavo, mañọívo, -iévo, mañaveámo, -aveáto, ma-

II. vadáivo, -óiva, -áivo, vadaveáma, -aveáto, vadévana.

III. partộiva, -óiva, -oóiva, partaveáms, -aveáto, partivons.

La $2^{2}$ I è modellata sulla forma corrispondente della II e III ${ }^{2}$; -avedmo dalla I alla II e III. ${ }^{3}$

Di ésso: ǵivo, ọios, óivo, aveáme, aveáto, évona; $-4^{2}$ e $5^{2}$ pure usate sono: saveáma, saveáta. Le prime forme sono analogiche sugli altri verbi, saveám e saveáto 4 sono analogiche su so, sáim ecc. ${ }^{5}$

\section{Perfetto.}

114. Modello per tutte le coniugaz.: Vadọivo o vodiẹtto, vadišto, vodétto, vodémmo, vodęsto, vodiérno. ${ }^{\circ}$ Rara è la $4^{2}$ vodęsamo; comune invece lá $3^{2}$ vadóisa. Altre forme in uso sono: Ia $3^{a}$ kan. deá; Ib $3^{2}$ allokuó; Ic $3^{2}$ mañié; III $3^{2}$ forni. ${ }^{6}$

Il $-v$ - della $I^{2}$ p. potrebbe credersi secondario col Meyer-Lübke II, 305; resta pero, ammettendo ciò, da spiegare come vi si introdusse, 7 chè la fonetica non soccorre. - I kandóivo $1^{2}$ p., invece di *kandiẹ́vo, è analogico sulla II e III; - -iẹtto <*-etti, onde il dittongo; -so di $3^{2}$ è congiunto al tema colla vocale $-\ell-$, onde -gi-; - - fsomo è sulla $3^{2}$-giso, se non piuttosto su diximus, e allo scempiamento di - ss- si giungerebbe pel $\$ 84.8$

115. Forme forti sono: in -u - vidda, $3^{2}$ vgllb; in -s- $3^{2}$ vólzo, vólzaro poco usati. Accanto a queste però ci son sempre le forme deboli. Di ésso: fuótto e fújo, fústo, fu; fuóssmo e fúmmo, fósto e

1 V. Ascoli AG. XV, 220 e altrove.

- Cfr. De Lollis AG. XII, 187; Parodi AG. XIII, 300.

- Cfr. Meyer-Lübke II, 290.

4 Cfr. Savini, 79 per Teramo; De Lollis AG. XII, 7 per Casalinc; e vedi pure Zingarelli 1. c., 235 .

5 E bene qui correggere l' opinione del Meyer-Lübke II, 289, ripetuta nella sintesi del Grundrifs, che a Foggia la $I^{a}$ è in -vu, e perciò raccostabile al $-v o$ toscano; il vero $e$ che la $I^{2}$ esce in $-v 2$, che potrebbe risalire a *-vu e $2 *$ - va.

- Cfr. l' antico lucchese in Salvioni, St. fil. rom. VII, 207, e l' arpinate in Parodi AG. XIII, 299.

7 Cfr. De Lollis, AG. XII, I89,

- Cfr. il reat. senţ̧ssemo. in Campanelli, 17. 
fústo, fürno e fuórno; nelle quali forme, come in tanta parte delle lingue neolatine, le basi fũ e fú sono l' una accanto all' altra.'

\section{Congiuntivo imperfetto.}

116. Modello per tutte le coniugazioni: vodésso, vodišso, vodéssa, vadassóima, vadassóita, vadíssara o -issona. La la ha pure kandásso $I^{2}$ e $3^{2}$ p.; la Ib anche allakkṕsso $1^{2}$ e $3^{2}$. Le con. I e III sono attratte dalla II.2 C' è pure la $4^{2}$ vodissomo, la $5^{2}$ vodisto, di uso molto ristretto però, e forse non indigene. ${ }^{3} \mathrm{Di}$ esss: fósso, fúuš, fọ́ssa, sassọimo, sassọito, fússara e fússono; sono pure in uso la $4^{2}$ fússsmo e fóssoma, la $5^{2}$ fústo e fósto. La $4^{2}$ e la $5^{2}$ in -óimo sono analogiche su sáimə, saveámə ecc. - L' imperft. è usato anche in funzione di pres. (cfr. § III).

\section{Participio presente.}

117. Resta assorbito dal gerundio. Ic, II, III fornęnna, vodęnno, mañęnno; Ia kandánna, Ib allakkónno. Le forme in -åndo, -ęndo sono agg. verb.

\section{Participio perfetto.}

118. Modelli: Ia kandeáto; Ib allokkuọto; Ic mañiéto, e fra questi della Ic vanno i part. dei verbi in -əję, che per le altre forme son passati alla II con. (cfr. § 106): sbrojéto, stozzajęto; - II e III aviuto, durmiuto, coll' attrazione della III alla II con., come generalmente nel Mezzogiorno d' Italia.

119. Forme forti sono: 10 in -t-: -gere slruitto, fritto, kjêndo pianto, tindo, slritto, arra $\widehat{g} g$ jind do, mindo; - -sc- nedto; - voc. + cdítto, kuoutio, fálto; cons. + c- tuórto; - -1- silldo scelto, kuóldo colto; - -p-, -b- skritto, rúlto, jindo empito; 4 -d- assindo sceso attratto da tîndo; - -r - apiẹ́rto, muọorto.

20. in -s-: -t-, -d- kjiuso, misso messo, róiso riso; - -ndstọiso steso, spọiso, dofóiso; - -rd- drso arso; - - $\mathrm{n}$ - arromedso; - -r - kúrzo corso; - -rg - spárzo sparso.

$3^{0}$. in -st-: arpuósto riposto, arrospuósto risposto, nnaskuósto nascosto, ssomuóstlo mosso, vísta, arramásti.

4\%. Residui da 'Itu: vivala bevuto e vissuto, arvivato ritornato a vita, kjuọvato piovuto.

Non di rado le forme deboli sono dal volgo preferite alle corrispondenti forti.

1 Cfr. Meyer-Lübke II, 340 sg.

Cfr. Savini, 68.

- Il Meyer-Lùbke II, 349 sg., dice che forme come vadassims ecc. son da considerare come neoformazioni, e di uso ristretto e limitato. Ad Agnone e nel territorio circostante invece queste forme sono le sole veramente volgari e generalmente diffuse. Ne sembra accettabile l' induzione dell' $A$. sulla priorità di vodtssomo ecc., induzione che per reggere ba bisogno di un' altra ipotesi non sicura.

- v. Salvioni, App. mer. $78 \mathrm{n}$.

Zeitschr f. rom. Phil. XXXIv. 


\section{Futuro.}

120. Modello di forme organiche:

$3^{2}$ fatarrd, $6^{2}$ fatarriénn. Sono in uso solo queste forme, e in senso dubitativo. ${ }^{1}$ Le forme sciolte $\mathrm{e} l$ ' ind. pr. sono usati per queste e per le altre persone.

\section{Condizionale.}

121. Modello per tutte le coniug.:

putarrọjjo e putgiro, putarrił̌š, putarrọjjo e putgiro, putarrissoma, putarrišto, putarríjons e putérons. Per la $4^{\mathrm{a}}$ e la $5^{2}$ si preferisce l' impft. cong. 2

Le forme provengono da habebam. Putgira, putẹ́rono sono reliquie del pcprft. -éra. ${ }^{3}$

$\mathrm{Di}$ ésso oltre a sarrọjjo ecc., c' è fẹ́ura $\mathrm{I}^{\mathrm{a}}$ e $3^{\mathrm{a}}$ per., fọ́rana, dal pcprft. suddetto.

\section{Appendice.}

\section{Saggio di versioni in agnonese.}

$$
\text { I. Il figliuol prodigo. }
$$

$N u$ banomo tonęva du fillo.

$R u$ kkju ćconínno dó́es' a rru pótra: nleáta, dámmo kẹllo ka

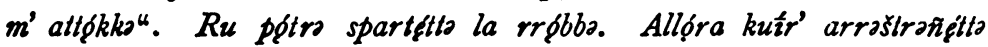
tútto kduso, \& ddǵppo na pikka do juórno, so no jętt' a nnu pajeso lundeána, \& llbko s' affrutted tuitto kauso, monánno na bbruitta vóito.

$K_{u}$ úndo nno $j$ armanqtio kkju kkubbęllo, jelto na piézzo do karoštóijo, lok' addó stedvo; o hisso, po nn' ave ke sso mantie, so motteso koe hiuno do kutro pajoiso, ko ro mannftt' a $\bar{g} \hat{g}$ yarded ro puórt' a lla massar jjj .

$S$ avrtja wultut' abbutted koe lla línno ka so mantfoono ro puórto, ma nosstiuno jo no dedvo.

Allór' arronźaviuto, doćgtto: „kudanda harkiun' a lla kedsa do pátromo sprẹkano lo pedna, e jji ękk mo moro do feáma." Mo tọngo la vójjo, vajj' a ppatrom' e jjo dọika: nteála, ájjo fátto pokkeáto kọndro do Ddọij” \&

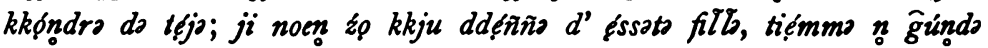
do hartéuno".

1 Cfr. D' Ovidio, AG. IV, $186 \mathrm{n}$.

- Non persuade quanto dice il Meyer-Lübke II, 365 , che cioè questa sostituzione sia di ragion fonetica sotto la spinta analogica dell' impft. cong., facendo p. e. andaressémo < andarssémo < andassémo.

3 Cfr. Parodi, AG. XIII, 302. 


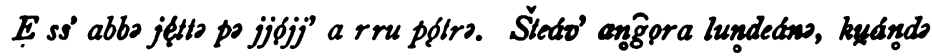

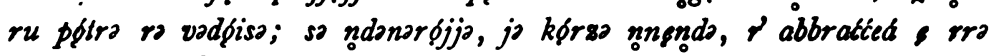
vašá. Ru fill jo dxiftto: "teáta, ájjo fatto pokkeato kóndro do Ddójj' \&

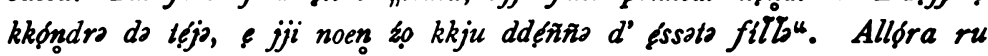
pótro doç̨t' a rro harżiuno: "Purtedto súbbot' $q k k$ ru vostito kkju mmello, \& mmattétajoro, e mmoltellaja l' anięll' a rru dóit' \& rro skárp' a

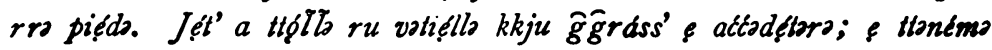
kummoito; pokké stu filto mójjo s' éva muórto, \& arsušted, s' toa pordiuta, \& ss' artruwed". E kkummonǵs'so ru fastbina. Ru fillo majüro

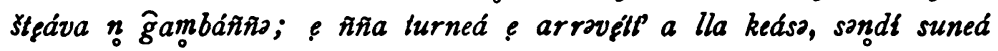
e bballedjo. Allọra kjamétto nu sorvotéuro, e jj' addummangiso ke tva kellla fessto; e kkutro j' arrospunnóiso: ne armoniuto frdtoto; eppdtroto a 'ćtíso ru votięllo kkju ğgrrásso, e tté kummóito, pokkẹ \& armontuto seán' e ssálv»". Ru majiuro se nğůjateá, \& nno wulẹva ndredjo. Ru pgtro seftt' \& rro kjameávo; \& hiss' arrospunnętt' \& ddoćs' a rru pótro: nékk, sp tánda hénno $k a j i$ to fáćc ru sorvotéura, e nnoem manívo mię móin' a ll' uórdono 'tójjo; \& a mme tu noen disto mié nu krapitto, po mmanfrmor? nsiémbra koe ro kumbentino mojjo. E mmeu, $\&$ armoniuto ssu fillo tójjo, dóppa ko s a 'ffruileáto la rróbba tájjo koe llo mealo fémmona, tu jẹ 'ctíso pa hisso ru votięllo kkju $\hat{g} \bar{g}$ rdsso.

E rru pótro ; arrospunnétto: „filt mójjo, tu stif sémbra koe mme, \& thutto lo mójj' \& llo tójjo. Ma s' éva tone kummoilt \& fea fostóino, pokké fratot' tva muórlo, e armonélt' a lla vóito, toa pordiuto, $f$ ss' artruwedjo"

\section{Novella IX della I giorn. del Decam.}

A rro tiémbo do ru pritmo rre de Cípro, dóppo ka Huffredo do

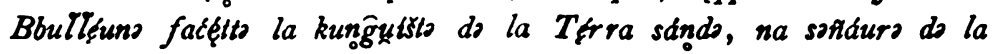

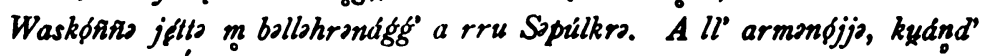
arrovelt' a C'Cipro, fós' akkjappeáto da ciérto medlo krostiẹ́no, ko jo ıutiẹrno l' unéuro. Hẹsso so rammarokcdvo, sẹnza putérzo kunżuledjo, \& pponked do ji a 'rkọrr' a rru rre. Ma na porsduno jo docéso ka sarrtjo

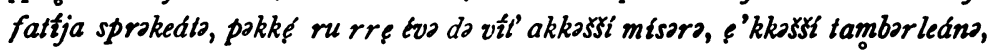
ko noen zulaméndo noen faćéva juštízojo do l' affẹso do l' éldro, ma so tulẹva piuro no zánda peáco ciẹndo milojo ko jo fatêvon' a hisso. A ssondt kęsto, kella fémmono pordétto la spordnza do la vonnétto; \& ppo kkunziularso n gadkka mangiro do la péna sájjo, panked do frossoje la samotidono do ru rre. - So no jétto kjantinno nnęind' a hisso, \& jjo dofétto: nsontéura, ji nno viénğa nnénd' a tte, $p$ ' avé vannélto do la med- 


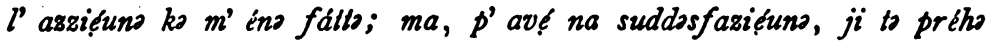
do mo nzañie na fié a ssuffri l' affrindo ko to fen' a tlejo. Akkušrz mo putésss mbareá piuro jójj' a ssuffri m baciénło ru weajjo mójjo! $E$ ss' $i$ lo putésso feá, lo sa Ddójjo ña to ro déra koe ttútto ru kéuro, na vólda ka tu si tándo wuớn' a ssuffrójjo."

Ru rrẹ, prím' Eva steáto liệnd' e spuldrọino, ma alléuro, ña so fọss' arrośboltiẹto da nu suọnno, facéso paheá keáro la meál' azziẹun

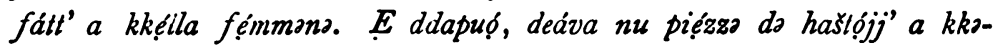
juinḡa facéva kakkáuso kọndr' a ll' unọ́ro do la kuróna sájjo.

GIOVANnI Ziccardi. 